\title{
Monte Carlo approach for studying microphases applied to the axial next-nearest-neighbor Ising and the Ising-Coulomb models
}

\author{
Kai Zhang (张凯) ${ }^{1}$ and Patrick Charbonneau ${ }^{1,2}$ \\ ${ }^{1}$ Department of Chemistry, Duke University, Durham, North Carolina 27708, USA \\ ${ }^{2}$ Department of Physics, Duke University, Durham, North Carolina 27708, USA \\ (Received 7 February 2011; revised manuscript received 17 April 2011; published 9 June 2011)
}

\begin{abstract}
The equilibrium phase behavior of microphase-forming systems is notoriously difficult to obtain because of the extended metastability of their modulated phases. In this paper we present a systematic simulation methodology for studying layered microphases and apply the approach to two prototypical lattice-based systems: the three-dimensional axial next-nearest-neighbor Ising (ANNNI) and Ising-Coulomb (IC) models. The method involves thermodynamically integrating along a reversible path established between a reference system of free spins under an ordering field and the system of interest. The resulting free-energy calculations unambiguously locate the phase boundaries. Simple phases are not found to play a particularly significant role in the devil's flowers and interfacial roughening plays at most a small role in the ANNNI layered regime. With the help of generalized order parameters, the paramagnetic-modulated critical transition of the ANNNI model is also studied. We confirm the $X Y$ universality of the paramagnetic-modulated transition and its isotropic nature.
\end{abstract}

DOI: 10.1103/PhysRevB.83.214303

PACS number(s): 64.60.Cn, 64.60.F-, 05.10.Ln, 75.10.-b

\section{INTRODUCTION}

Lattice models are central to statistical mechanics. They strip away the complexity due to packing and help reveal the influence of nongeometrical factors on both equilibrium and nonequilibrium self-assembly. The Ising model, for instance, offers a singular window on critical phenomena and on gas-liquid coexistence; ${ }^{1}$ Flory-Huggins's theory of solvated polymers is core to the physics of polymers; ${ }^{2}$ and spin glasses are key sources of inspiration for the difficult problem of structural glass formation. ${ }^{3}$ If a lattice model of a system exists, it is often a good strategy to solve it before embarking on a study of more elaborate variants.

Microphase formation is one such phenomenon that could benefit from further consideration of lattice-based models. The frustration of short-range attraction-or sometimes repulsion ${ }^{4}$ - by a long-range repulsion, irrespective of the physical and chemical nature of these interactions, leads to universal spatially modulated patterns. ${ }^{5}$ Periodic lamellae, cylinders, clusters, etc., are thus similarly found in block copolymers, ${ }^{6-8}$ oil-water surfactant mixtures, ${ }^{9,10}$ charged colloidal suspensions, ${ }^{11}$ and numerous magnetic materials. ${ }^{12,13}$ Microphase formation has also been hypothesized to play a role in biological membrane organization ${ }^{14}$ and in the formation of stripes in certain superconductors, ${ }^{15-20}$ although the microscopic interpretation is still debated. The spontaneous nature of microphase organization allows for these mesoscale periodic textures to find technological success as thermoplastic elastomers ${ }^{6}$ and nanostructure templates. ${ }^{21}$

Obtaining detailed control over microphase morphology remains, however, notoriously difficult. ${ }^{22}$ Annealing, ${ }^{23}$ external fields, ${ }^{24}$ strain compression, ${ }^{25}$ addition of fullerenes, ${ }^{8,26}$ or complex chemical environments ${ }^{27}$ are often necessary to order diblock copolymers, for instance. Understanding how to tune and stabilize microphases is essential to broadening their material relevance, yet experimental systems provide limited microscopic insights. A number of continuous space ${ }^{28-34}$ and lattice $^{35-43}$ models have thus been devised for theoretical and simulation studies, some of which have even become textbook material. ${ }^{44,45}$ Grasping the equilibrium properties of these models is necessary to resolve problems surrounding the nonequilibrium assembly of microphases. ${ }^{46-48}$ But although the modulated regime is a key feature of these models, it has not been accurately characterized in any of them. Even for the most schematic formulations, the existing theoretical treatments have only offered limited assistance.

Direct computer simulations have also been unable to provide reliable equilibrium information. ${ }^{45,49}$ Traditional simulation methodologies that facilitate ergodic sampling of phase space by passing over free-energy barriers, notably parallel tempering and cluster moves, are of limited help in microphase-forming systems. Because of the dependence of the equilibrium periodicity on temperature, sampling higher temperatures leaves the system in a modulated phase with the wrong periodicity; and because of the lack of simple structural rearrangements for sampling different modulations, the efficiency of cluster moves is limited. We recently introduced a free-energy integration method for simulating modulated phases that overcomes this hurdle. ${ }^{50}$ Here, we detail this method and apply it to the study of two canonical three-dimensional (3D) spin-based systems: the axial nextnearest-neighbor Ising (ANNNI) and the Ising-Coulomb (IC) models. Both of these models are known to form lamellar phases of different periodicities at low temperature, but their phase structure is still not completely understood. The phase information we obtain by simulation further allows testing of various theoretical predictions. The plan of this paper is to introduce the models (Sec. II), the simulation methodology (Sec. III), and the generalized order and critical parameters (Sec. IV). After discussing the results (Sec. V), a short conclusion follows.

\section{MODELS}

Before introducing the models, a clarification of the nomenclature for describing layered microphases is in order. 

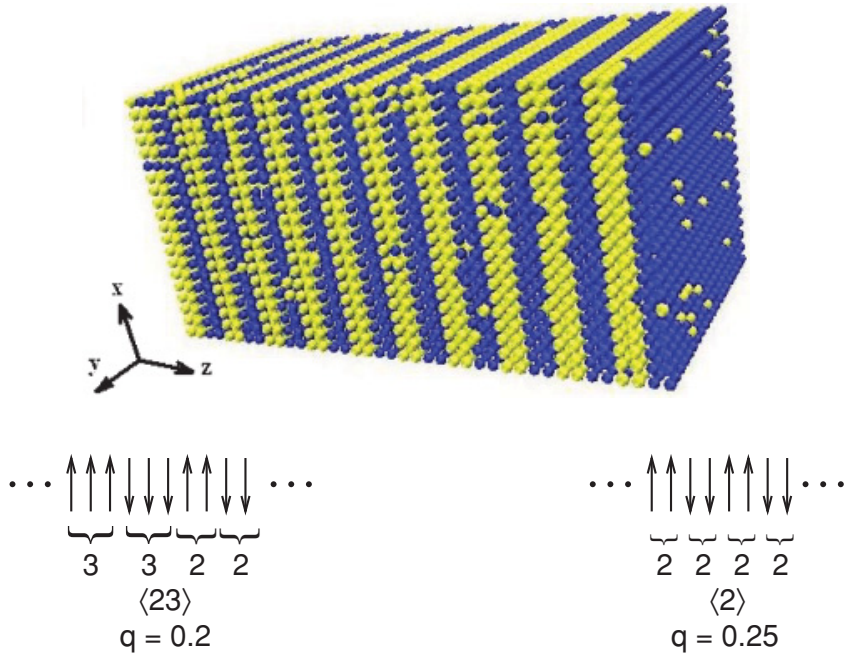

FIG. 1. (Color online) (Top) Snapshot of the ANNNI antiphase $\langle 2\rangle$ at $T=2.4, \kappa=0.7$ for a $20 \times 20 \times 40$ lattice. Differently shaded beads indicate spins up or down. (Bottom) Notation examples of the lamellar phases $\langle 23\rangle$ and $\langle 2\rangle$.

Two conventions for characterizing the periodicity of lamellar phases coexist in the scientific literature. The first compactly identifies a phase with a simple wave number $q=1 / \lambda$ (in units of $2 \pi$ ), where $\lambda$ is the period length. The second, a short-hand form $\left\langle m^{j} n^{k}\right\rangle$ introduced in Ref. 51, is less compact but provides a more intuitive description of the layered phase. In this notation, integers are used to describe a lamellar phase formed by periodic repetition of patterns of $j$ pairs of opposite-spin lamellae of width $m$ followed by $k$ pairs of opposite-spin lamellae of width $n$ (Fig. 1). For example, phase $\langle\infty\rangle$ is the ferromagnetic phase and phase $\langle 2\rangle$ consists of two layers of spins up followed by two layers of spins down. But phase $\langle 23\rangle$ has a period $\lambda=5$, because thermal fluctuations blur the layer boundaries and the thickness of each lamella takes the average value

$$
\frac{\lambda}{2}=\frac{m j+n k}{j+k}
$$

Although this notation, can only represent phases of rational periodicity, it is well suited for describing the commensurate phases that are here observed.

\section{A. ANNNI model}

The ANNNI model was first introduced to rationalize helical magnetic order in certain heavy rare-earth metals. ${ }^{36-38,52}$ The simple model's description of the experimentally observed order is only qualitative, ${ }^{53}$ but because of its surprisingly complex phase behavior, it is now canonical for the study of systems with competing interactions. ${ }^{44,45}$ Its Hamiltonian on a simple cubic lattice

$$
H_{\mathrm{ANNNI}}=-J \sum_{\langle i, j\rangle} s_{i} s_{j}+\kappa J \sum_{[i, j]_{z}} s_{i} s_{j}
$$

is expressed for spin variables $s_{i}= \pm 1$ coupled through a positive constant $J$. With the Boltzmann constant $k_{B}$, $J / k_{B}$ sets the temperature $T$ scale. Alignment is favored for nearest-neighbor pairs $\langle i, j\rangle$ but frustrated with relative strength $\kappa>0$ for $z$-axial next-nearest-neighbor pairs $[i, j]_{z}$. The exact solution of the one-dimensional version of the model provides $T=0$ phase information for all other dimensions: ${ }^{51}$ Ferromagnetic order is the ground state for $\kappa<1 / 2$, while the layered antiphase $\langle 2\rangle$ minimizes the energy for $\kappa>1 / 2$. A mean-field description qualitatively captures the higher dimensional, finite- $T$ features of the model ${ }^{54,55}$ The system is paramagnetic at high $T$; it is ferromagnetic at low $T$ and $\kappa$; and modulated layered phases form for sufficiently high $\kappa^{38}$. These three regimes join together at a multicritical Lifshitz point $\left(\kappa_{L}, T_{L}\right)$ whose special critical properties have been predicted by theory ${ }^{56-58}$ and verified in simulations. ${ }^{59,60}$ Hightemperature series expansions have also been used to study the paramagnetic phase and predict its limit of stability ${ }^{61,62}$ These predictions were confirmed by finite-size critical rescaling for the paramagnetic-ferromagnetic (PF) transition ${ }^{51,63-65}$ and by heat capacity ${ }^{51,66}$ and generalized susceptibility ${ }^{50}$ measurements for the paramagnetic-modulated (PM) transition. For $\kappa<\kappa_{L}$, the PF transition has Ising universality, ${ }^{65,67}$ while for $\kappa>\kappa_{L}$, the PM transition has been argued to have $X Y$ universality ${ }^{36,68,69}$ but direct simulation verifications are incomplete $^{50}$ and the results of the high-temperature series expansion analysis are inconclusive. ${ }^{62,70}$ The ferromagneticmodulated (FM) transition is predicted by a Landau-Ginzburg treatment to be first order with $q$ changing discontinuously from 0 , and to be tangent to the PF and PM transition lines at the Lifshitz point. $^{71}$

Commensurate $\left\langle 2^{j} 3\right\rangle$ phases spring from the multiphase point at $T=0$ and $\kappa=1 / 2$. The structure of the branching processes at low $T$ has been carefully studied ${ }^{72}$ and forms the basis for the low-temperature series expansion. ${ }^{73}$ For the rest of the modulated regime, approximate theoretical treatments, such as a mean-field theory with a soliton correction, ${ }^{74}$ an effective-field theory, ${ }^{75}$ and the tensor product variational approach (TPVA) ${ }^{76}$ have been used. Monte Carlo simulations have also been carried out in this regime, ${ }^{51,64}$ but the hysteresis resulting from the high free-energy barriers that separate modulated phases from each other limits accurate determinations of the phase boundaries from annealing-based approaches. ${ }^{37,50}$ Avoiding annealing is thus preferable for accurately locating transitions within the modulated regime..$^{50}$ It is thought that incommensurate phases and interfacial roughening could lower the transition free-energy barriers between different commensurate modulated phases on sufficiently large lattices ${ }^{72}$ but these phases have not been observed thus far.

\section{B. Ising-Coulomb model}

The Ising-Coulomb (IC) model, in which the nearestneighbor ferromagnetic coupling spin is frustrated by longrange Coulomb interaction of relative strength $Q$, was first suggested as a model for the stripe-phase behavior of high-temperature superconductors in two dimensions. ${ }^{42,77}$ It was also adopted as a generic coarse-grained description of microphase formation in systems with competing pair interactions in three dimensions $\mathrm{s}^{43,78,79}$ and used to study the effect of dispersion forces on phase transitions in ionic 
systems. ${ }^{80}$ Although it is based on the Ising model, its Hamiltonian,

$$
H_{\mathrm{IC}}=-J \sum_{\langle i, j\rangle} s_{i} s_{j}+Q J \sum_{i>j} \frac{s_{i} s_{j}}{r_{i j}},
$$

does not allow ferromagnetic ordering for any $Q>0$. An infinitesimally small Coulomb frustration is sufficient to induce layering. ${ }^{78}$ By analogy with a Landau-Ginzburg model with frustration, ${ }^{42,77,81,82}$ it is, however, expected that any screening of the Coulomb interaction moves the onset of modulation to a finite $Q \cdot{ }^{83,84}$ Interestingly, the $Q \rightarrow \infty$ limit recovers the simple-cubic lattice restricted primitive model (LRPM) of Dickman and Stell at full occupancy. ${ }^{85,86}$

The one-dimensional $T=0$ phase sequence is known to be made of equal-length blocks of alternating orientation. ${ }^{87} \mathrm{In}$ higher dimensions, although no rigorous demonstration exists, layered phases of integer periodicity are also expected to be the ground state at low $Q .{ }^{79}$ In that regime, an approximate mapping to a one-dimensional system seems reasonable. For sufficiently large $Q$, two- and three-dimensional periodic structures, i.e., "cylinders" and "clusters," minimize the energy, and for $Q>Q_{N}^{0} \approx 15.33$ antiferromagnetic Néel order is expected. ${ }^{79}$ Mean-field treatments ${ }^{79,80}$ and Monte Carlo simulations (for $Q<1)^{43}$ describe the paramagneticmodulated (PM) transition. Although the mean-field treatment overestimates the transition temperature, ${ }^{79,80}$ its predictions are generally similar to the phase behavior obtained from simulations. ${ }^{43}$ Because of the long-range isotropic Coulomb interaction, the transition is "fluctuation-induced" first order for any $0<Q<Q_{N},{ }^{88,89}$ and at low $Q$ the modulated phases melt at $T_{c}(Q) \sim T_{c}(0)-Q^{1 / 4}$, where $T_{c}(0) \approx 4.51$ is the $3 \mathrm{D}$ Ising simple cubic critical point. ${ }^{78}$ For $Q \geqslant Q_{N}$ the continuous paramagnetic-Néel (PN) transition has Ising universality, and at high $Q$, the critical temperature $T_{c}(Q) \sim T_{c}(\infty) Q-$ $T_{c}(0),{ }^{79}$ where the trivial linear dependence results from the choice of units and $T_{c}(\infty) \approx 0.515$ is the LRPM result. ${ }^{85,90} \mathrm{~A}$ triple point connects the paramagnetic, modulated cluster, and antiferromagnetic Néel phases at $\left(Q_{N}, T_{c}\left(Q_{N}\right)\right)$, but only the mean-field estimates $Q_{N}=36 / \pi \approx 11.5$ and $T_{c}\left(Q_{N}\right)=1.61$ are known. ${ }^{79}$ Within the modulated layered regime proper, phases spring out at the boundary between neighboring low-temperature ground states of integer periodicity. ${ }^{43}$ The process is akin to that observed between the antiphase and the ferromagnetic phase in the ANNNI model. The simulations in the layered regime capture the presence of these phases, but the use of a simulated-annealing approach in a strongly hysteretic regime is likely to have biased the estimates for the transition temperatures. ${ }^{43}$

\section{METHOD}

Monte Carlo simulations are here used for determining the absolute free energy of the different modulated phases. The thermodynamic integration, the reference systems, and the Monte Carlo sampling details are presented in this section.

\section{A. Thermodynamic integration}

The free energy is obtained from Kirkwood thermodynamic integration, ${ }^{91,92}$ which involves simulating a system with a
Hamiltonian that couples a reference system Hamiltonian $H_{0}$ with that of the system of interest $H_{1}$ :

$$
H_{\lambda}=(1-\lambda) H_{0}+\lambda H_{1} .
$$

For a given $\lambda$, the Helmholtz free energy $F_{\lambda}$ obeys

$$
\frac{\partial F_{\lambda}}{\partial \lambda}=-\frac{T}{Z_{\lambda}} \frac{\partial Z_{\lambda}}{\partial \lambda}=\left\langle\frac{\partial H_{\lambda}}{\partial \lambda}\right\rangle_{\lambda},
$$

where $Z_{\lambda}$ is the canonical partition function and $\langle\cdots\rangle_{\lambda}$ denotes a canonical average under $H_{\lambda}$. The difference between the free energy of the system of interest $F_{1}$ and that of a known reference system $F_{0}$ at phase point $\left(T_{0}, \kappa_{0}\right)$ is thus

$$
\begin{aligned}
F_{1}\left(T_{0}, \kappa_{0}\right)-F_{0}\left(T_{0}, \kappa_{0}\right) & =\int_{0}^{1}\left\langle\frac{\partial H_{\lambda}}{\partial \lambda}\right\rangle_{\lambda} d \lambda \\
& =\int_{0}^{1}\left\langle H_{1}-H_{0}\right\rangle_{\lambda} d \lambda .
\end{aligned}
$$

In order to obtain reliable numerical results, the integration path from $\lambda=0$ to 1 must be reversible; no first-order phase transition may take place along it. The choice of reference system, which is central to the approach, is detailed in the next subsection. The numerical integration is done by simulating the system at discrete $\lambda$ points chosen following a Gauss-Lobatto scheme. ${ }^{93}$ Because of a rapid change in the integration curve as $\lambda \rightarrow 1$, the latter part of the integral uses logarithmically spaced points that are densely distributed near $\lambda=1$ (Fig. 2). This adjustment is necessary for accurately sampling the $z$-axis lattice translations of small systems in weak external fields.

Investigating the $T$-frustration plane point by point is computationally wasteful. Data acquisition is significantly accelerated by thermally integrating to nearby temperatures $T_{1}$ or frustrations $\kappa_{1}$ (or, equivalently, $Q_{1}$ ) using a known state point $\left(T_{0}, \kappa_{0}\right)$ as reference by

$$
\frac{F_{1}\left(T_{1}, \kappa_{0}\right)}{T_{1}}-\frac{F_{1}\left(T_{0}, \kappa_{0}\right)}{T_{0}}=\int_{T_{0}}^{T_{1}}\left(\frac{\partial F_{1}}{\partial 1 / T}\right)_{\kappa_{0}} d(1 / T)
$$

or

$$
F_{1}\left(T_{0}, \kappa_{1}\right)-F_{1}\left(T_{0}, \kappa_{0}\right)=\int_{\kappa_{0}}^{\kappa_{1}}\left(\frac{\partial F_{1}}{\partial \kappa}\right)_{T_{0}} d \kappa,
$$

where

$$
\left(\frac{\partial F_{1}}{\partial 1 / T}\right)_{\kappa_{0}}=\left\langle H_{1}\right\rangle_{\kappa_{0}}
$$

and

$$
\left(\frac{\partial F_{1}}{\partial \kappa}\right)_{T_{0}}=\left\langle\frac{\partial H_{1}}{\partial \kappa}\right\rangle_{T_{0}}=\left\langle J \sum_{[i, j]_{z}} s_{i} s_{j}\right\rangle_{T_{0}} .
$$

In practice, the free-energy results are fitted with a polynomial of degree three or four. The free energy at any point within a relatively short interval is then interpolated from the parameterized function.

\section{B. Reference system}

In order to guarantee a reversible integration path, the reference system should reflect the symmetry of the phase 

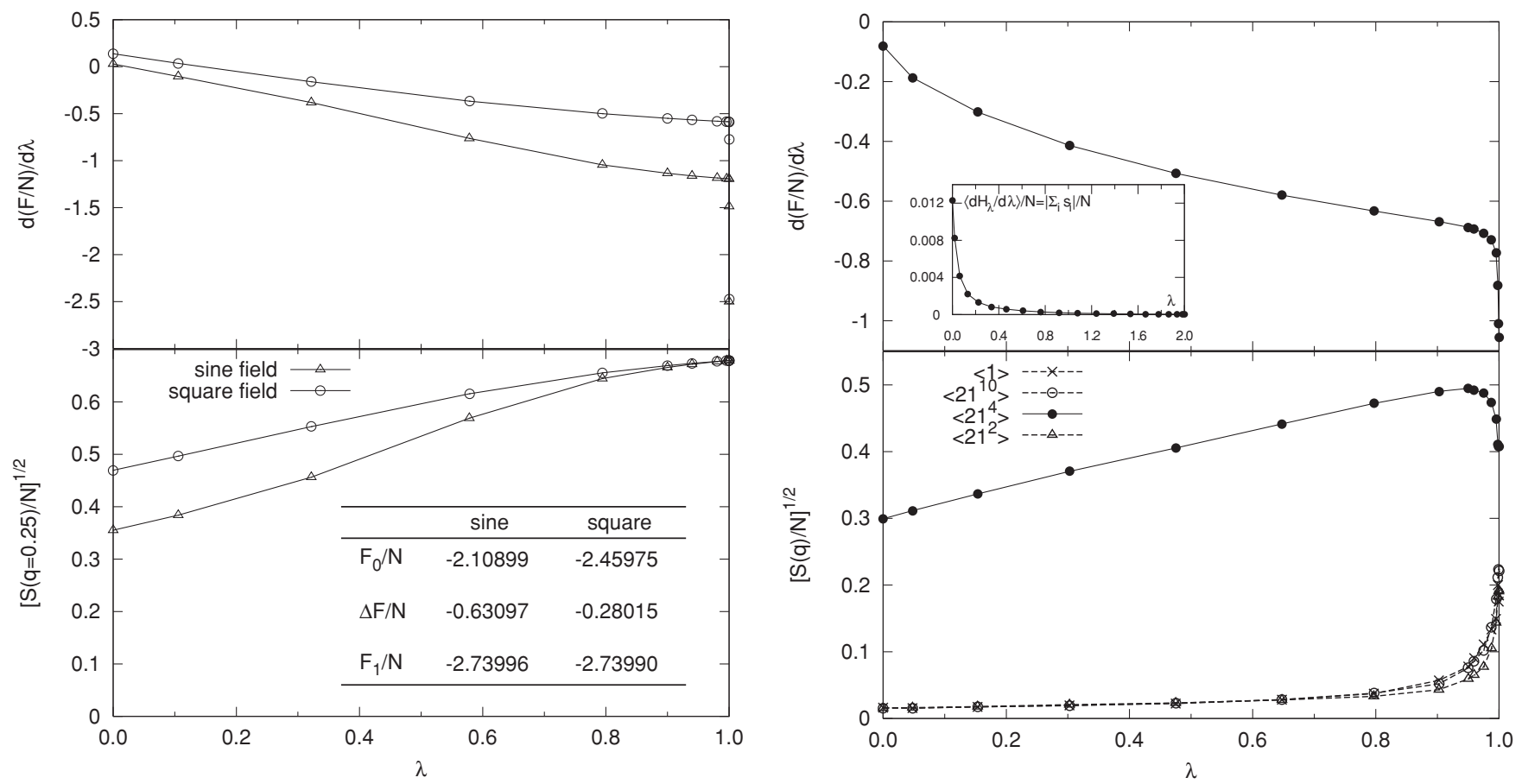

FIG. 2. (Top left) Thermodynamic integration of the ANNNI model at $\kappa=0.7$ and $T=2.5$ for phase $\langle 2\rangle$ using sinusoidal and square fields as reference. (Bottom left) Change in the structure factor peak height along the integration path and the free-energy results for the two different references. (Top right) Thermodynamic integration of the IC model at $Q=0.8$ and $T=1.06$ for phase $\left\langle 21^{4}\right\rangle$. The integration curve from a fluctuating to a constant magnetization system is shown in the inset. (Bottom right) The structure factor at different wave vectors demonstrates the preservation of the modulation along the integration path.

under investigation. A good reference system should also have a Hamiltonian $H_{0}$ whose partition function $Z_{0}$ and free energy $F_{0}$ can be obtained analytically or at least with high numerical accuracy. For the lamellar phases observed on lattices with $N=L_{x} L_{y} L_{z}$ sites, we propose a reference that has decoupled spins under a $z$-axial periodically oscillating field $B(z)$ with amplitude $B_{0}$,

$$
H_{0}=-B_{0} \sum_{i=1}^{N} s_{i} B\left(z_{i}\right),
$$

similarly to the periodic potential wells confining free particles used in Ref. 94. It trivially follows that,

$$
\frac{F_{0}}{N T}=-\frac{1}{L_{z}} \sum_{z=1}^{L_{z}} \ln \left[2 \cosh \left(\frac{B_{0} B(z)}{T}\right)\right] .
$$

The amplitude $B_{0}$ should be sufficiently strong to prevent layer melting and changes of layer periodicity as the field is turned off, yet sufficiently weak to allow sampling of the integrand. ${ }^{95}$ Fortunately, the relatively high free-energy barriers between neighboring modulated phases make phase transitions along the integration path highly unlikely, even if sections of the path are formally metastable. Due to the broken symmetry between the different coordinate axis, we can also, without loss of generality, lock the lamellae in a specific orientation when initializing configurations for the IC model.

The applied field $B(z)$ need not be the exact equilibrium profile of the modulated layers as long as the integration from $B(z)$ can be done reversibly. For instance, either square or sinusoidal fields can be used as reference states for the study of modulated phases with integer periodicity. The free-energy results of both approaches agree with high accuracy (Fig. 2). The equivalence also holds in the low-temperature regime, where the ground-state profile is more akin to a square well than to a pure sine function. ${ }^{51}$ Because sinusoidal fields are "soft" in the interlayer region, which helps averaging the layer fluctuations, and because they provide a compact and efficient way to describe noninteger periodic lamellae, we choose

$$
B(z)=\sin \left(2 \pi q z+\phi_{0}\right),
$$

where a small phase angle $\phi_{0}$ is added to prevent the lattice planes from directly overlapping with the zeros of the field.

The IC model, which must remain charge neutral, requires that the reference partition function $Z_{0}$ be computed subject to a fixed magnetization constraint. In the infinite system limit this correction is negligible, but on a finite lattice it may affect transition temperatures. For the paramagnetic phase, the reference system Hamiltonian $H_{0}$ with $B_{0}=0$ results in $F_{0} / N=-T \ln 2$ for an unconstrained system [Eq. (12)], but the properly constrained reference system instead has

$$
\frac{F_{0}^{\prime}}{N T}=-\frac{1}{N} \ln \left(\begin{array}{c}
N \\
N / 2
\end{array}\right),
$$

where $\left(\begin{array}{c}N \\ N / 2\end{array}\right)$ is the binomial coefficient. For $N=12^{2} \times 24=$ 3456 spins, the difference between the two results is $\sim 0.001 T$, which may be significant because of the small entropy differences between layered phases. Calculating $F_{0}^{\prime}$ is not, 
however, as straightforward for $B_{0} \neq 0$. One has to define a thermodynamic integration path between fluctuating and constant magnetization systems

$$
H_{\lambda}=H_{0}+\lambda\left|\sum_{i} s_{i}\right|,
$$

with $\lambda$ going from 0 to $\infty$. In practice, $\left\langle\frac{\partial H_{\lambda}}{\partial \lambda}\right\rangle=\left|\sum_{i} s_{i}\right|$ rapidly decays to zero with growing $\lambda$, and therefore integrating to a finite $\lambda$ of order unity is sufficient. The zero-magnetization free energy $F_{0}^{\prime}$ is then obtained by adding the correction from thermodynamic integration to $F_{0}$ from Eq. (12) (Fig. 2). We note, however, that even in the small IC systems studied here, the free-energy corrections for different modulated phases are very similar for a given temperature. The phase transitions are thus only imperceptibly affected by the shift.

\section{Monte Carlo sampling}

We perform constant- $T$ Monte Carlo (MC) simulations on a cubic lattice under periodic boundary conditions, using $N=L_{x} L_{y} L_{z}=40^{2} \times 240$ spins for the ANNNI model and $N=12^{2} \times 24$ spins for the IC model, unless otherwise noted. Ewald summation is used to compute the long-range Coulomb interactions in the IC model..$^{92,96}$ The phases studied have wave numbers $q=n / L_{z}$ with integer $n$ 's, which keeps modulations commensurate with the lattice. We initialize the modulated phases with a sinusoidally varying spatial probability of the desired periodicity. We find that the system relaxes to the equilibrium spin profile for a given $x y$ plane,

$$
s_{x y}(z) \equiv \frac{1}{L_{x} L_{y}} \sum_{i \in x y}\left\langle s_{i}\right\rangle,
$$

irrespectively of the initialization scheme as long as it has the correct periodicity.

Basic MC sampling consists of single-spin flips for the ANNNI model. Spin exchanges, which enforce charge neutrality, are used for the IC model. Phase-space exploration gains in efficiency by complementing the basic sampling with iterations that take advantage of phase symmetry.

(1) For the modulated phases, layer swaps allow for the individual layer thickness to fluctuate while preserving the overall periodicity. Multiple layer swaps are necessary to alter the periodicity and therefore even neighboring modulated phases are well separated in configuration space.

(2) Near the PM transition, an anisotropic cluster algorithm for the ANNNI model ${ }^{59}$ and a modified Wolff algorithm that considers the corrections from long-range interaction for the IC model ${ }^{43}$ are used, in order to capture the strong fluctuations.

(3) For systems with an applied external magnetic field, random displacements of the entire modulated pattern relative to the field along the $z$ axis help sample the translational degrees of freedom.

For reference point integrations, up to $10^{5} \mathrm{MC}$ moves $(\mathrm{N}$ attempted spin flips or exchanges per move) are performed after $5 \times 10^{4} \mathrm{MC}$ moves of preliminary equilibration. For the thermal and frustration integrations, only $10^{4} \mathrm{MC}$ moves are necessary, because the free energy is not as sensitive to the accuracy of the integration slope as it is to its starting point over the small $T$ intervals considered. In the vicinity of the critical transitions, we also use the multiple histogram algorithm, in order to obtain high-precision results with a minimal number of computations. ${ }^{97}$ The method relies on reweighing the sampled configurations at a fixed temperature $T_{0}$, typically nearby $T_{c}$, by the Boltzmann factor difference $e^{-\left(1 / T-1 / T_{0}\right) E}$ for results at neighboring temperatures $T .{ }^{98}$ Our implementation uses a logarithmic summation scale, in order to avoid sum overflow in large systems. ${ }^{98}$

\section{ORDER AND CRITICAL PARAMETERS}

Structural order parameters help locate phase transitions and are particularly important for the study of continuous and weakly first-order transitions in models studied here. The generalization and application of the study of critical and roughening transitions in modulated phases is presented in this section.

\section{A. Modulation order parameters}

Functions of the Fourier spin density

$$
\tilde{s}_{q} \equiv \sum_{i=1}^{N} s_{i} e^{i 2 \pi q z_{i}}
$$

are natural choices for characterizing modulations in layered systems. The simplest of them, the generalized magnetization per spin, is defined analogously to the absolute magnetization in the Ising model $^{43}$

$$
\begin{aligned}
\langle m(q)\rangle & =\frac{1}{N} \sqrt{\left\langle\tilde{s}_{q}\right\rangle\left\langle\tilde{s}_{-q}\right\rangle} \\
& =\frac{1}{N} \sqrt{\left\langle\sum_{i} s_{i} \cos \left(q z_{i}\right)\right\rangle^{2}+\left\langle\sum_{i} s_{i} \sin \left(q z_{i}\right)\right\rangle^{2}} .
\end{aligned}
$$

A direct use of $\langle m(q)\rangle$, however, causes problems in long simulations, because in principle it averages to zero as the lattice drifts along the $z$ direction, which eventually recovers translational symmetry (Fig. 3). Maximizing the real component of $\tilde{s}_{q}$ with respect to a phase shift in the $z$ direction for each configuration before taking the thermal average resolves this issue. In practice, we use a straightforward parabolic interpolation scheme. ${ }^{99}$ Using the optimized version of $\langle m(q)\rangle$, even in simulations that are too short for the system's periodicity to completely diffuse, significantly improves the data quality (Fig. 3). Only quantities based on optimized $\tilde{s}_{q}$ are therefore used in the rest of this work. The generalized magnetization decays $\langle m(q)\rangle \sim\left(T_{c}-T\right)^{\beta}$ with critical exponent $\beta$, but the decay properties are not ideal for numerically detecting critical temperatures in finite systems.

The next higher magnetization moment, the $z$-axial static structure factor, is similar to the equivalent liquid-state quantity

$$
\begin{aligned}
N S(q) & \equiv\left\langle\tilde{s}_{q} \tilde{s}_{-q}\right\rangle=N^{2}\left\langle m^{2}(q)\right\rangle \\
& =\left\langle\left|\sum_{i} s_{i} \cos \left(q z_{i}\right)\right|^{2}+\left|\sum_{i} s_{i} \sin \left(q z_{i}\right)\right|^{2}\right\rangle,
\end{aligned}
$$

where $\left\langle m^{2}(q)\right\rangle$ is the second moment of the magnetization. ${ }^{51,61}$ Both $S(q)$ and its normalized version $\sqrt{S(q) / N}=\sqrt{\left\langle m^{2}(q)\right\rangle^{43}}$ grow upon cooling and are maximal at the wave number $q_{c}$ 


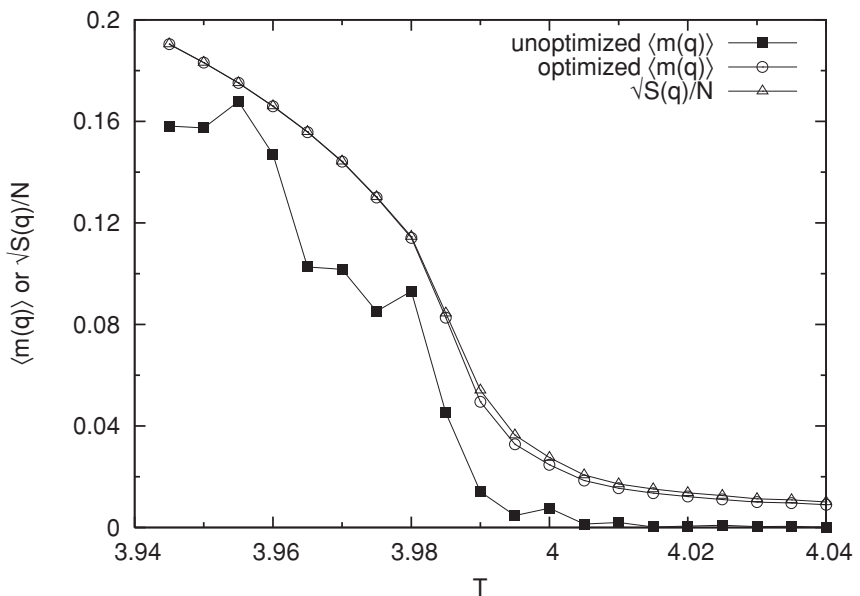

FIG. 3. Optimized and unoptimized $\langle m(q)\rangle$ and $\sqrt{S(q) / N}$ for the ANNNI model at $\kappa=0.7$ and $q_{c}=0.1917$. The difference between $S(q) / N$ and $\langle m(q)\rangle^{2}$ gives $\chi(q)$ [Eq. (20)].

of the first modulated phase below $T_{c}$. The monotonically increasing $S(q)$ is, however, ill-suited for detecting the PM transition in simulations, because, like $\langle m(q)\rangle$, it does not give a clear visual signature of $T_{c}$. The generalized susceptibility

$$
\begin{aligned}
T \chi(q) & \equiv \frac{1}{N}\left(\left\langle\tilde{s}_{q} \tilde{s}_{-q}\right\rangle-\left\langle\tilde{s}_{q}\right\rangle\left\langle\tilde{s}_{-q}\right\rangle\right) \\
& =N\left\langle m^{2}(q)\right\rangle-N\langle m(q)\rangle^{2},
\end{aligned}
$$

i.e., the second cumulant of the magnetization, does not suffer from this caveat. It indeed diverges on both sides of the transition $\chi\left(q_{c}\right) \sim\left|T-T_{c}\right|^{-\gamma}$ with critical exponent $\gamma$, as would $\chi(0)$ in the Ising model, and was used in our previous study ${ }^{50}$ (Fig. 4). But directly correcting for finite-size effects results in a high sensitivity of the transition location to simulation noise. The Binder-cumulant route is more convenient for detecting $T_{c}$, because its value at the critical point $U_{4}^{*}$ is straightforwardly insensitive to scaling the system size. ${ }^{45,100}$ For layered phases, a generalization of the expression

$$
U_{4}(q)=1-\frac{\left\langle m^{4}(q)\right\rangle}{3\left\langle m^{2}(q)\right\rangle^{2}}
$$

in terms of the second and the fourth $\left\langle m^{4}(q)\right\rangle=\left\langle\tilde{s}_{q}^{2} \tilde{s}_{-q}^{2}\right\rangle / N^{4}$ $q$-modulated magnetization moments is needed.

Because of the anisotropy of the modulated phases, it is useful to review how breaking isotropy may affect critical properties. In a system of dimensions parallel $L_{\|} \equiv L_{z}$ and perpendicular $L_{\perp} \equiv L_{x}=L_{y}$ to the modulation propagation, the correlation length $\xi$ may diverge with different critical exponents

$$
\xi_{\|} \sim\left|T-T_{c}\right|^{-v_{\|}}, \quad \xi_{\perp} \sim\left|T-T_{c}\right|^{-v_{\perp}} .
$$

The critical Binder cumulant $U_{4}^{*}=U_{4}\left(q_{c}, T_{c}\right)$ is then invariant for a fixed ratio $L_{\|} / L_{\perp}^{\nu_{\|} / \nu_{\perp}}$ (Fig. 5). ${ }^{101,102}$ At a uniaxial Lifshitz point, such as in the ANNNI model, ${ }^{59} v_{\|} \simeq \frac{1}{2} v_{\perp} \cdot{ }^{56,57}$ For the PM transition, at $\kappa>\kappa_{L}$, we also consider the possibility of anisotropic critical behavior. Although a direct determination of $v_{\|} / v_{\perp}$ is numerically difficult, indirect finite-size study of systems with a fixed ratio $L_{\|} / L_{\perp}=2$ shows that $U_{4}^{*}$ does not

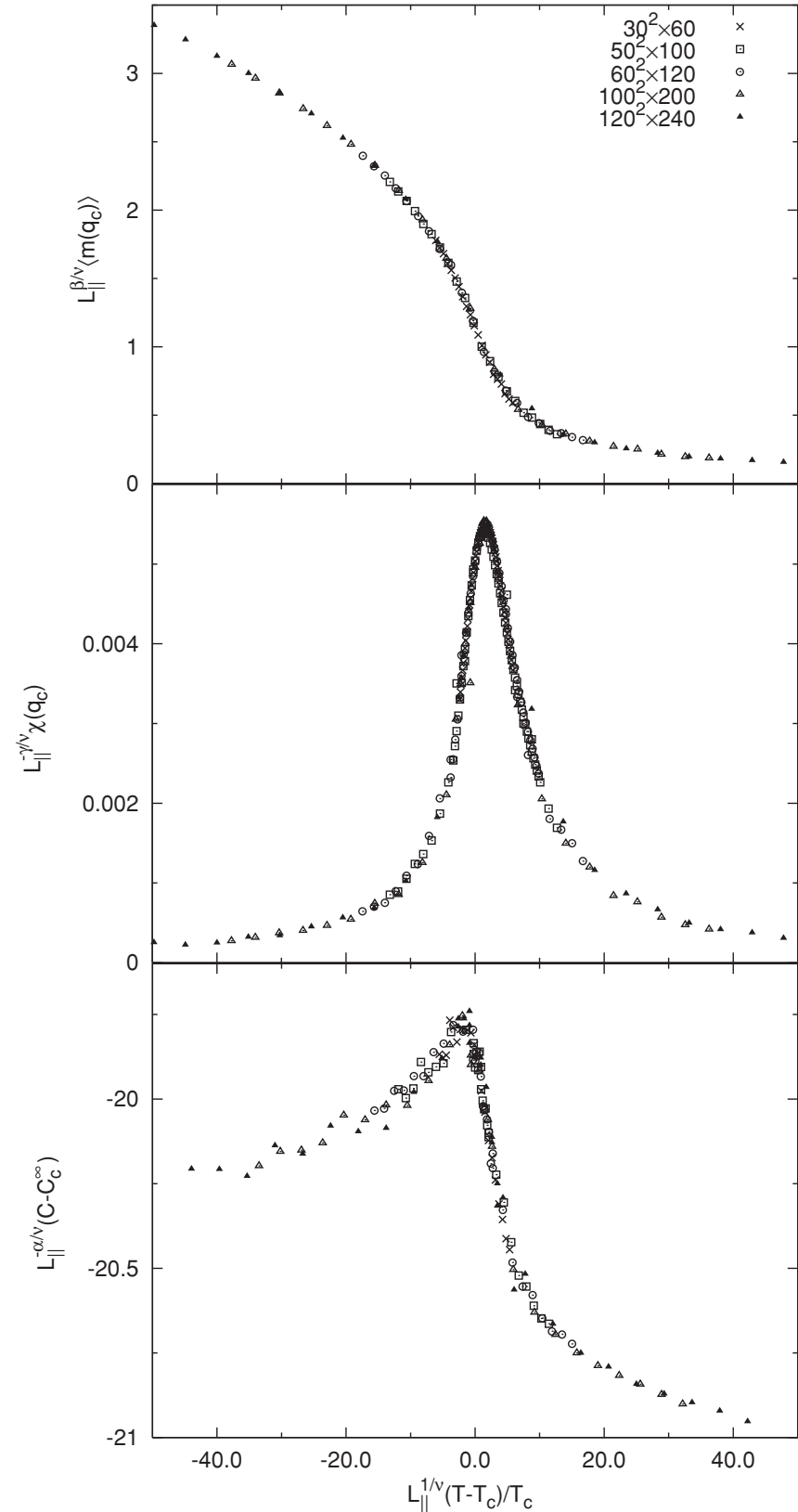

FIG. 4. Finite-size scaling of the magnetization (top), susceptibility (middle), and heat capacity (bottom) of the ANNNI model at $\kappa=$ 0.8 and $q_{c}=0.2$, using $v=0.66, \gamma=1.32, \beta=0.34, \alpha=-0.01$, and $C_{c}^{\infty}=20$.

vary at the $\mathrm{PM}$ transition (Fig. 5). This observation suggests that $\nu_{\|} / \nu_{\perp} \approx 1$; i.e., $\xi_{\|}$and $\xi_{\perp}$ diverge with the same critical exponent $v_{\|}=v_{\perp} \equiv v$ at the PM transition. Critical anisotropy is thus neglected in the rest of this study.

Binder cumulants also allow us to independently determine the critical exponent $v$ using the peak value of the derivative of $U_{4}$ : 


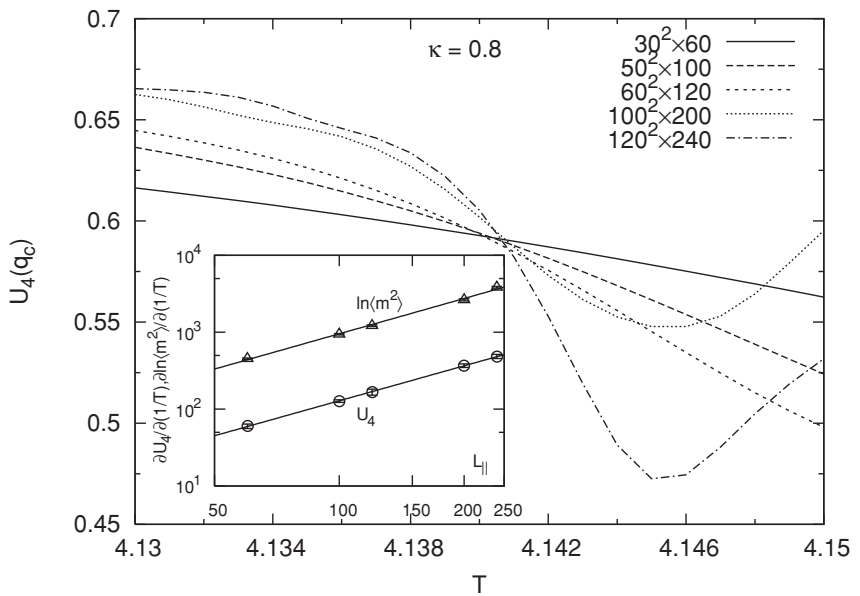

FIG. 5. Binder cumulant of the ANNNI model at $\kappa=0.8$ and $q_{c}=0.2$. The curves, which intersect at the critical temperature $T_{c}=4.141$, monotonically decrease with $T$. The limited validity regime of histogram reweighing results is here responsible for the nonmonotonicity. (Inset) Finite-size scaling analysis of the peak of the derivative of $U_{4}\left(q_{c}\right)$ and $\ln \left\langle m\left(q_{c}\right)^{2}\right\rangle$. The logarithm scales as $1 / v$, which here gives $v=0.66(2)$.

A similar relation for the structure factor gives ${ }^{45,97}$

$$
\ln \left(\frac{\partial \ln \left\langle m^{2}(q)\right\rangle}{\partial 1 / T}\right)_{\max }=\frac{1}{v} \ln L+\text { constant. }
$$

The system size $L$ in the scaling relation can be either $L_{\|}$ or $L_{\perp}$ as long as the ratio $L_{\|} / L_{\perp}$ is fixed. For an isotropic critical point, as long as the dimensions are rescaled by the same factor, the form of the collapse and the critical exponents remain unchanged (Fig. 5).

Once $v$ and $T_{c}$ are obtained, the critical exponents $\beta$ and $\gamma$ can more easily be determined through finite-size scaling. ${ }^{45}$ The quantities $L^{\beta / v}\left\langle m\left(q_{c}\right)\right\rangle$ and $L^{-\gamma / v} \chi\left(q_{c}\right)$ overlap for different system sizes when drawn as a function of the scaled temperature $L^{1 / v}\left(T-T_{c}\right) / T_{c}$. The heat capacity can also be similarly rescaled, but only if $C$ diverges at $T_{c}$, as for transitions with Ising universality. For transition with $X Y$ universality, for which $\alpha=-0.01, C$ peaks at a finite value $C_{c}^{\infty}$ in the infinite system size limit. The proper scaling relation is then $L^{-\alpha / \nu}\left(C-C_{c}^{\infty}\right),{ }^{103,104}$ but such rescaling for a small $\alpha$ is subject to sizable numerical errors. ${ }^{103}$ The hyperscaling relation $2-\alpha=3 v$ is used instead to determine $\alpha{ }^{105}$

\section{B. Interfacial roughening}

In the Ising ferromagnetic regime, even though the correlation length isotropically diverges at the critical point, the interface between two regions of opposite magnetization presents a roughening transition $T_{R}$ at roughly half the critical temperature. ${ }^{106-109}$ Below $T_{R}$ the interface is localized, while above $T_{R}$ its width diverges logarithmically with surface area. In simulation, an interface is created within the bulk by using an antiperiodic boundary condition along the $z$ direction ${ }^{106}$ and the transition can be localized by finite-size analysis (Fig. 6). Modulated phases present a series of interfaces between lamellae of opposite magnetization that may also roughen with increasing temperature. It has indeed been

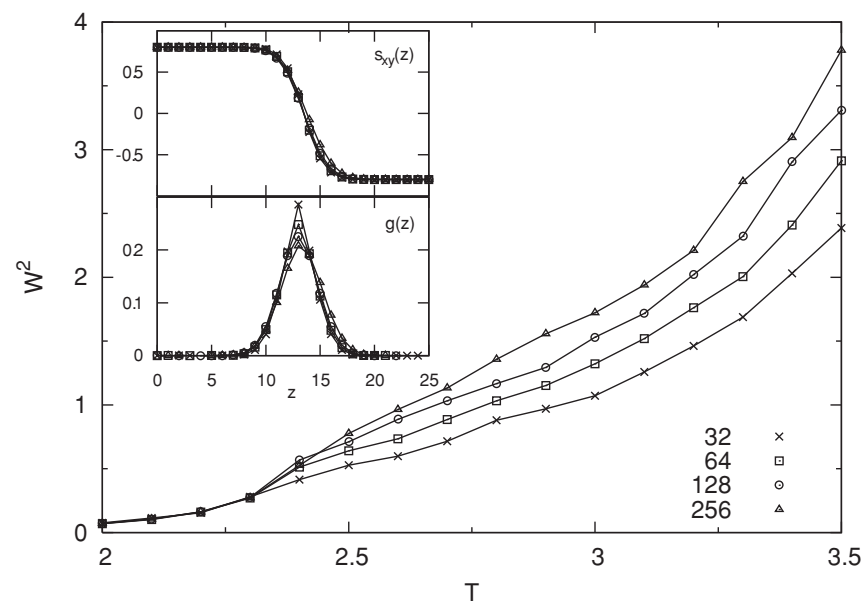

FIG. 6. The roughening transition in the ferromagnetic regime at $\kappa=0.2$ using systems of size $L^{2} \times 26$ is found at $T_{R}=2.35(5)$. (Insets) Average magnetization profile and gradient at $T=3.5$.

suggested that the interface location of modulated lamellae should logarithmically diverge. ${ }^{88,110}$ If that were the case, interlayer fluctuations at temperatures between $T_{R}$ and $T_{c}$ could participate in phase branching and the formation of equilibrium incommensurate structures in the large-system limit. $^{111}$

A generalization of the simulation approach is necessary to study this question. The variance of the interface position $z$,

$$
W^{2} \equiv\left\langle(z-\langle z\rangle)^{2}\right\rangle
$$

measures the fluctuations of the interface location and is expected to diverge logarithmically with system size for $T>T_{R}$

$$
W^{2} \sim \ln L_{\perp}
$$

For $T<T_{R}, W^{2}$ should have an even weaker system size dependence. For thermal averaging, the normalized magnetization gradient

$$
\begin{aligned}
g(z) & =\frac{d s_{x y}(z) / d z}{\int\left[d s_{x y}(z) / d z\right] d z} \\
& =\frac{s_{x y}(z+1)-s_{x y}(z)}{\sum_{z}\left[s_{x y}(z+1)-s_{x y}(z)\right]},
\end{aligned}
$$

is used as a weight function. ${ }^{112}$ The equilibrium profile $s_{x y}(z)$ is obtained by aligning instantaneous profiles to correct for lattice drift before averaging (Fig. 6). For the modulated regime, where multiple interfaces are present, layers within half a period of the interface $i$, i.e., layers whose $z$ coordinates belong to a set $I$, are grouped together in the variance calculation

$$
W_{i}^{2}=\sum_{z \in I} z^{2} g(z)-\left(\sum_{z \in I} z g(z)\right)^{2},
$$

and the results for the various interfaces are averaged at the end. 


\section{RESULTS AND DISCUSSION}

Assembling the results from the various observables obtained from the computational techniques provides a clearer understanding of the equilibrium phase behavior of the ANNNI and IC models. In this section, we concentrate on the properties of the modulated regime.

\section{A. Phase transitions between layered phases}

The size of the energy gap between neighboring phases with $q$ 's commensurate with the simulation box reflects the limited and constrained choice of modulations realizable on a finite periodic lattice (Fig. 7). In an infinite periodic system, where all rational modulations are valid but irrational $q$ 's are excluded, this gap would be infinitely small because rational numbers are dense on the real axis. ${ }^{36,64,113}$ The smooth and extended energy curves for the different modulations are also characteristic of strongly metastable phases (Figs. 7 and 8 ). The high free-energy barriers between layers of differing periodicity result in phases that are sufficiently long-lived to persist throughout the entire simulation if the
$L_{x} L_{y}$ cross section is large enough. Different phases can be observed at a given temperature and frustration, depending on how the system is initialized. For smaller cross sections, however, the reduced number of spins involved in changing the periodicity lowers these transition barriers. For a fixed system size, although a longer $L_{z}$ allows the study of more modulated phases, the need to keep these phases stable limits the maximal aspect ratio $L_{\|}: L_{\perp}$ of the simulation lattice. In practice, the selected ratio must balance these competing demands. A microscopic understanding of the transition mechanism between layered phases of different periodicity is still incomplete, ${ }^{51}$ but we empirically find that a size ratio of $2: 1$ is sufficient.

The crossing of the free-energy curves for neighboring modulated phases identifies the transition temperature. Using this approach side steps the hysteresis that otherwise afflicts annealing approaches and results in a more accurate depiction of the modulated regime than has previously been obtained. ${ }^{43,63,64,114}$ For the IC model, for instance, the freeenergy calculations locate the phase transitions at temperatures at least $10 \%$ lower than reported in Ref. 43, where the the
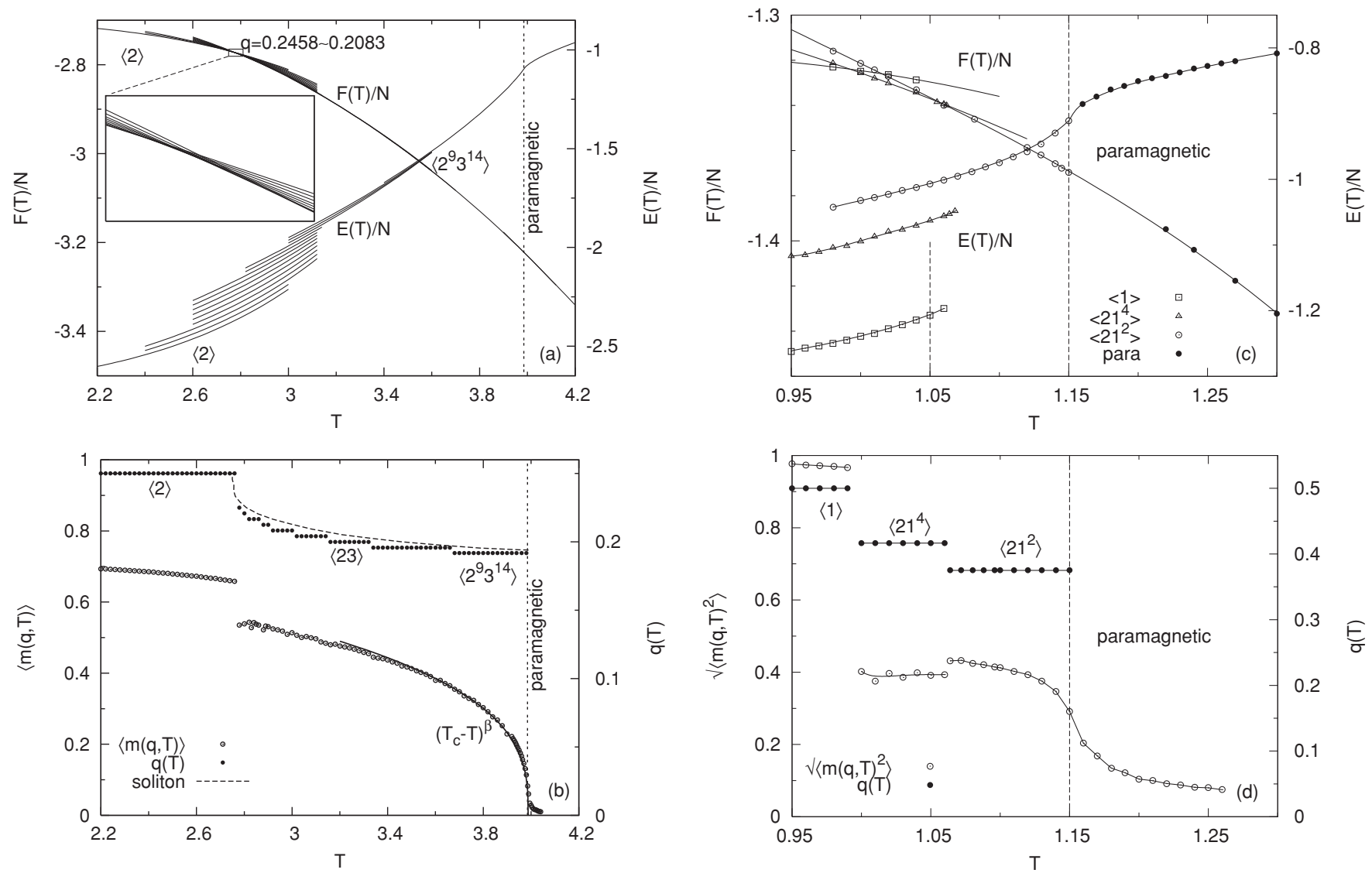

FIG. 7. (a) Energy and free-energy results for the ANNNI model at $\kappa=0.7$ for modulations ranging from phase $\langle 2\rangle$ to phase $\left\langle 2^{9} 3^{14}\right\rangle$ at melting. The PM transition $T_{c}=3.988$ (vertical dashed line) is obtained from $U_{4}^{*}$. (b) Under the same conditions as (a), equilibrium devil's staircase compared with the rescaled soliton result (dashed line), and $\langle m(q)\rangle$ compared with the power-law decay form with $\beta=0.34$ obtained from finite-size scaling (solid line). Note that in the low-temperature limit, the square profile of phase $\langle 2\rangle$ gives $\langle m(q)\rangle \rightarrow 2^{-1 / 2}$. (c) Energy and free-energy results for the IC model at $Q=0.8$ for phases $\langle 1\rangle,\left\langle 21^{4}\right\rangle$, and $\left\langle 21^{2}\right\rangle$, and the paramagnetic phase. The PM transition $T_{c}=1.15(1)$ is obtained from $C$. The short vertical dashed line indicates the transition temperature between phases $\langle 1\rangle$ and $\left\langle 21^{2}\right\rangle$, obtained from simple annealing (see text; Ref. 43). (d) Under the same conditions as (c), devil's staircase and normalized structure factor. Note that in the low-temperature limit, the profile of phase $\langle 1\rangle$ gives $\sqrt{\left\langle m(q)^{2}\right\rangle} \rightarrow 1$. 


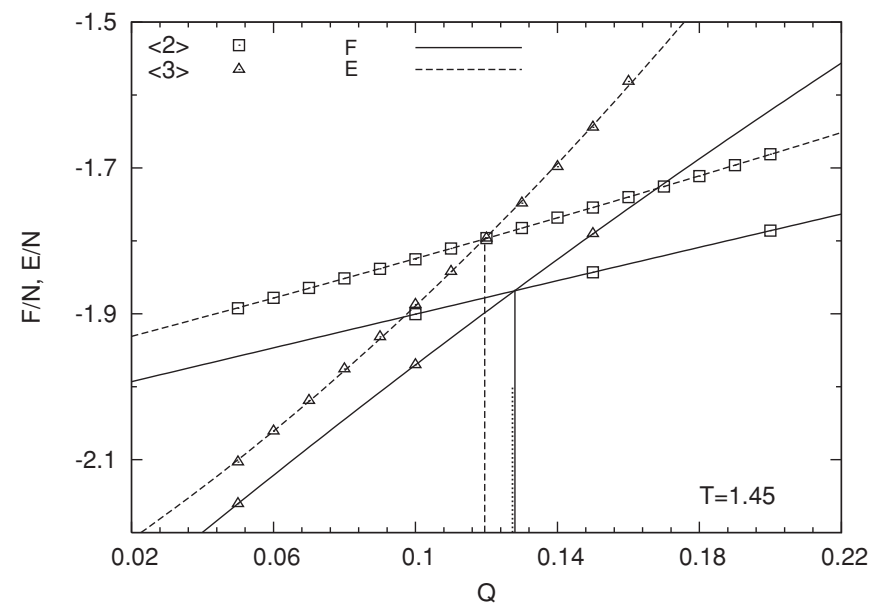

FIG. 8. Energy and free-energy curves of the IC model for phases $\langle 3\rangle$ and $\langle 2\rangle$ at $T=1.45$. The phase boundary from free-energy calculation (solid lines) agrees with the $T=0$ mean-field prediction (dotted line) and is different from the energy inversion (dashed lines; Ref. 79).

system was prepared in the $T=0$ ground state and studied by simulated annealing. At $Q=0.8$, we can even identify a commensurate modulated phase $\left\langle 21^{4}\right\rangle$ that was entirely missed by the annealing study. The other possibly missed commensurate phase $\left\langle 21^{10}\right\rangle$ is, however, unstable here as well, presumably because of finite-size effects (Fig. 7). Qualitatively similar results are also obtained for $Q=0.144$ and $Q=0.17$ (not shown).

By integrating over frustration at low temperature we can also identify the boundary between phases of integer periodicity in the IC model. The results at finite temperatures agree very closely with the $T=0$ energy derived transitions. The location of the free-energy crossover between phases $\langle 1\rangle$ and $\langle 2\rangle$ (not shown) as well as between phases $\langle 2\rangle$ and $\langle 3\rangle$ is only mildly affected by temperature (Fig. 8). The thermal fluctuations produce a similar free-energy shift of both phases, which leaves the $Q$ location of the transition unchanged. We thus expect similar results at other $\langle n\rangle-\langle n+1\rangle$ phase transitions.

The PM transition is not accurately obtained by direct freeenergy comparisons for either system. For the ANNNI model, the continuous transition is best studied through the specialized tools of critical phenomena (see below). But even for the IC model, the fluctuation-induced first-order transition does not result in sufficiently high free-energy barriers for noticeably supercooling the paramagnetic phase in such a small system. The system instead rapidly freezes into a modulated phase below the transition and shows only a minimum of hysteresis. As a result, the transition identified from the heat capacity peak by annealing in Ref. 43 is equivalent to what is obtained here. A more careful system size dependence study would be necessary to refine the transition estimate.

\section{B. Devil's staircase and order parameter}

The equilibrium wave number obtained from the freeenergy results displays the characteristic devil's staircase. ${ }^{74}$ The stability regime of a given modulated phase stretches over an ever smaller $T$ range upon cooling. For the ANNNI model, the predicted truncation of the sequence before reaching the antiphase makes the staircase "harmless,"115 but the simulated system size is here insufficient to distinguish this scenario from the infinite "devil's last step" sequence in which no commensurate phase is missed. ${ }^{36,115}$ The overall shape of the decay can, however, be compared with the soliton theory prediction. ${ }^{74}$ Although the soliton does not correctly capture the PM transition temperature, once $T$ is linearly rescaled to make $T_{c}$ coincide, the agreement is fairly good (Fig. 7).

The equilibrium generalized magnetization behaves similarly to its $q=0$ version in the Ising model. For the ANNNI model around $T_{c}$, the quantity grows monotonically upon cooling. It continuously increases at first, but jumps discontinuously upon reaching the antiphase. In this last region the magnetization profile tends toward a periodic square, whose profile structure is only partially captured by a simple sinusoidal function. For the IC model, the renormalized structure factor, which is indistinguishable from $\langle m(q)\rangle$ at low
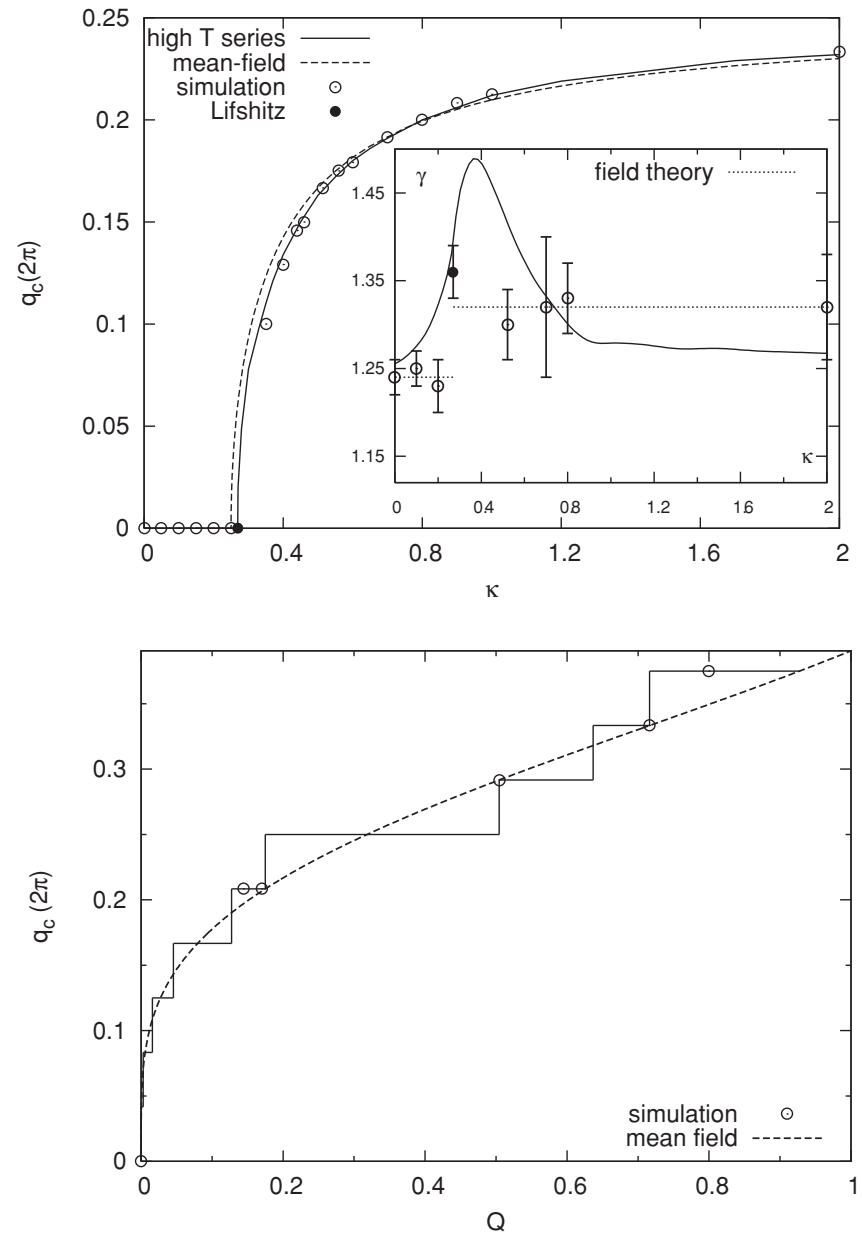

FIG. 9. Simulation wave number periodicity at melting $q_{c}$ for different frustration strengths of the ANNNI (top) and the IC (bottom) models superimposed with theoretical predictions. The solid line for the IC model captures the accessible wave number for $L_{z}=24$. (Top inset) The critical exponent $\gamma$ obtained by finite-size scaling is compared with the high-temperature series expansion (Ref. 62) and the field-theory predictions for the exponent (Refs. 68 and 69). The Lifshitz information is taken from Ref. 59. 
temperatures, is also not an ideal order parameter. When the system changes from phase $\left\langle 21^{2}\right\rangle$ to $\left\langle 21^{4}\right\rangle$, for instance, the peak height actually goes down. Here again, the modulation profile is not well captured by a simple sinusoidal function. In both models, the inclusion of higher order harmonics might better detect growing order upon cooling.

\section{Modulation at melting}

The periodicity of the modulated phase at the PM transition $q_{c}(\kappa)$ [or $q_{c}(Q)$ ] is remarkably insensitive to the theoretical approach used for capturing its behavior. In the ANNNI model, the agreement between simulation results, mean-field theory, 55 HT series expansion, ${ }^{61,62}$ and the critical scaling near the Lifshitz point

$$
q_{c} \sim\left|\kappa-\kappa_{L}\right|^{\beta_{l}},
$$

using the critical exponent either from series expansion $\beta_{l}=$ $0.5 \pm 0.05^{61,62}$ or from renormalization group $\beta_{l}=0.514,{ }^{117}$ is very good (Fig. 9). The similarity of the RG critical exponent with the mean-field value further suggests that the dependence of microphase periodicity on frustration is much easier to capture than the transition temperature. The free-energy correction due to fluctuations is likely similar for neighboring layered phases.

For the IC model, the mean-field prediction for the continuously changing $q_{c}$ is also within the simulation accuracy in the layered regime (Fig. 9), but the relatively small lattice size prevents a quantitative assessment of the theoretical predictions. In the high- $Q$ regime, where a Néel-paramagnetic-modulated phase triple point is expected, our coarse simulation estimate $Q_{N} \approx 15.8$ clearly differs from the mean-field prediction of 11.5. ${ }^{79}$ A large deviation between the theoretical prediction and the direct calculation is also observed at $T=0$, where $Q_{N}^{0}=9.549$ and 15.33 , respectively. ${ }^{79}$ Both those differences can mostly, and possibly completely, be explained by the low accuracy of the lattice Fourier transform in the large- $Q$ limit, where modulated phases of small domains form. ${ }^{79}$ Note, however, that the critical nature of the $Q_{N}$ point, which depends on the properties of the modulated-Néel transition, could also impact its location. If it is a bicritical point, fluctuations could result in larger deviations from the mean-field predictions. A generalization of the free-energy simulation approach to other modulated geometries should be able to resolve this question, but is beyond the scope of this work.

\section{ANNNI critical behavior}

The critical properties of the ANNNI model have been extensively studied using a high-temperature (HT) series expansion. ${ }^{61,62,70}$ For instance, critical temperatures can be estimated by resumming truncated series with Padé approximants. ${ }^{62,118}$ The arbitrariness of selecting the Padé order results in a range of estimates (Tables I and II). The values of $T_{c}$ obtained from finite-size scaling quantitatively agree with these estimates and are an order of magnitude more precise than both the HT series results and previous simulation estimates. ${ }^{65,66}$

The critical exponents from the HT series expansion, however, only qualitatively agree with the simulation results. The coefficients of HT series change continuously with $\kappa$. Finite series can thus only smoothly approximate sudden changes in critical behavior, while the critical exponents are here expected to change discontinuously on both side of the Lifshitz point. The apparent continuous variation of $\gamma$ with $\kappa$ obtained from HT series expansion is thus unphysical and should disappear if an infinite number of expansion terms were used. ${ }^{61}$ Field theory arguments suggest, however, that the critical exponents should have Ising universality below the Lifshitz point, $X Y$ above the Lifshitz point, ${ }^{68}$ and uniaxial Lifshitz universality at the Lifshitz point. ${ }^{57}$ The Ising ${ }^{65}$ and Lifshitz point ${ }^{59}$ predictions have been previously confirmed by Monte Carlo simulations, but above the Lifshitz point the model's behavior is not so clear. In particular, the HT series results for $\gamma$ at large $\kappa$ undershoot the $X Y$ exponent value. In the words of Ref. 62, at high $\kappa$ "a puzzling and unexplained feature [of the HT series expansion results] is the apparent decrease of $\gamma$ to something like the Ising value." Later similar studies did not quite resolve this question and even suggested that a different type of universality might be observed beyond $\kappa \approx 2 .{ }^{70}$ Our earlier simulation results did not provide a clear resolution of this issue either, because of limited system sizes and insufficient averaging in the critical region. ${ }^{50}$ Simulation of larger systems using the multiple-histogram method lifts any remaining ambiguity. The critical exponent results support an $X Y$ universality of the transition for all $\kappa>\kappa_{L}$ studied. The values of $v$ and $\gamma$ agree with each other and with the

TABLE I. Critical parameters of the ANNNI model for $\kappa \leqslant \kappa_{L}=0.270(4)$ obtained by finite-size scaling of cubic systems with $L=$ $16,32,40,64,80$ for $\kappa=0.1$ and 0.2 and from previous simulations (Refs. 59 and 65). Ising values are given for reference. The uncertainty on $T_{c}$ and $q_{c}$ from the HT series expansion results from the Padé-approximant method (Ref. 62). At $\kappa_{L}, v_{\|}$is reported. The starred $\left(^{*}\right) \alpha$ results are obtained from the hyperscaling relation $3 v=2-\alpha$ (or $\alpha+2 \beta+\gamma=2$ for $\kappa=0.24)$.

\begin{tabular}{llllllll}
\hline \hline$\kappa$ & Ising & 0.1 & $0.15^{\mathrm{a}}$ & 0.2 & $0.24^{\mathrm{a}}$ & $0.265^{\mathrm{a}}$ & $0.270^{\mathrm{b}}$ \\
\hline$T_{c}^{\mathrm{MC}}$ & 4.512 & $4.265(1)$ & $4.15(2)$ & $3.987(1)$ & $3.86(2)$ & $3.77(2)$ & $3.7475(5)$ \\
$T_{c}^{\mathrm{HT}}$ & $4.51(2)$ & $4.26(2)$ & $4.13(2)$ & $3.98(2)$ & $3.85(2)$ & $3.75(2)$ \\
$\nu$ & 0.63 & $0.62(1)$ & $0.61(3)$ & $0.62(2)$ & & $0.51(4)$ & $0.47(12)^{*}$ \\
$\alpha$ & 0.11 & $0.14(3)^{*}$ & $0.17(9)^{*}$ & $0.14(6)^{*}$ & $0.28(12)^{*}$ & $0.18(2)$ \\
$\beta$ & 0.34 & $0.31(2)$ & $0.30(3)$ & $0.31(3)$ & $0.23(3)$ & $0.19(2)$ & $1.40(6)$ \\
$\gamma$ & 1.24 & $1.25(2)$ & $1.20(6)$ & $1.23(3)$ & $1.26(6)$ & $1.36(3)$ \\
\hline \hline
\end{tabular}

${ }^{\mathrm{a}}$ Reference 65 .

${ }^{\mathrm{b}}$ Reference 59. 
TABLE II. See Table I for details. Critical parameters of the ANNNI model for $\kappa>\kappa_{L}$ obtained by finite-size scaling of systems with $L_{z}=60,120,150,180$, and 240 at $\kappa=0.522, L_{z}=120,240$, and 360 at $\kappa=0.7, L_{z}=60,100,120,200$, and 240 at $\kappa=0.8, L_{z}=60,120,180$, and 240 at $\kappa=2.0$, and from previous simulations (Refs. 51 and 66). $X Y$ values are given for reference.

\begin{tabular}{|c|c|c|c|c|c|c|c|}
\hline$\kappa$ & $X Y^{\mathrm{a}}$ & $0.5^{\mathrm{b}}$ & 0.522 & $0.6^{\mathrm{c}}$ & 0.7 & 0.8 & 2.0 \\
\hline Phase & & $\langle 3\rangle$ & $\langle 3\rangle$ & $\left\langle 2^{9} 3^{34}\right\rangle$ & $\left\langle 2^{9} 3^{14}\right\rangle$ & $\langle 23\rangle$ & $\left\langle 2^{6} 3\right\rangle$ \\
\hline$q_{c}^{\mathrm{MC}}$ & & $0.17(3)$ & $0.167(4)$ & $0.18(3)$ & $0.192(4)$ & $0.200(4)$ & $0.233(4)$ \\
\hline$q_{c}^{\mathrm{HT}}$ & & $0.162(1)$ & $0.167(1)$ & $0.180(1)$ & $0.192(1)$ & $0.200(1)$ & $0.232(1)$ \\
\hline$q_{c}^{\mathrm{MF}}$ & & 0.1667 & 0.1705 & 0.1816 & 0.1919 & 0.1994 & 0.2301 \\
\hline$T_{c}^{\mathrm{MC}}$ & 2.202 & $3.6(1)$ & $3.723(1)$ & $3.82(3)$ & $3.988(1)$ & $4.141(1)$ & $5.796(1)$ \\
\hline$T_{c}^{\mathrm{HT}}$ & $2.202^{\mathrm{d}}$ & $3.67(2)$ & $3.72(2)$ & $3.81(2)$ & $3.99(3)$ & $4.14(1)$ & 5.79(1) \\
\hline$v^{c}$ & 0.67 & & $0.66(2)$ & & $0.67(4)$ & $0.66(2)$ & $0.67(3)$ \\
\hline$\alpha$ & -0.01 & & $0.02(6)^{*}$ & & $-0.01(9)^{*}$ & $0.02(6)^{*}$ & $-0.01(9)^{*}$ \\
\hline$\beta$ & 0.35 & & $0.35(2)$ & & $0.35(3)$ & $0.34(3)$ & $0.36(2)$ \\
\hline$\gamma$ & 1.32 & & $1.30(4)$ & & $1.32(8)$ & $1.33(4)$ & $1.32(6)$ \\
\hline
\end{tabular}

${ }^{\mathrm{a}}$ Reference 105 .

${ }^{\mathrm{b}}$ Reference 66 .

${ }^{\mathrm{c}}$ Reference 51 .

${ }^{\mathrm{d}}$ Reference 116.

$X Y$ values, and most are significantly different from the Ising exponents (Table II and Fig. 9). The finite-size scaling of $C$ using $\alpha$ derived from hyperscaling relations further supports the agreement (Fig. 4). These observations additionally shed some doubt on the existence of a transition at $\kappa \approx 2 .^{70}$

The $X Y$ universality of the PM transition can be understood from the similarity between its two-component order parameter and that of the $X Y$ model. ${ }^{119,120}$ A mean-field picture for the order parameter of the ANNNI model [Eq. (19)] suggests that a spin $i$ in the modulated phase can be thought of as evolving within a magnetization profile of periodicity $q$ formed by all the other spins. A change of the average local magnetization at position $i$ is equivalent to shifting the phase angle $q z_{i}$ with respect to that profile. Note that the isotropic nature of the critical PM transition also suggests that pairs of spins parallel and perpendicular to the $z$ axis are equivalently correlated. The $z$-axis magnetization profile itself is correlated in the $x$ and $y$ directions. In the language of $X Y$ model, the phase angle is also correlated under translations in the $x$ or $y$ directions.

Why then, one may wonder, do the series expansion results not converge to the $X Y$-type $\gamma$ value at high $\kappa$ ? Examining the limit $\kappa \rightarrow \infty$ suggests an answer. In that limit the nextnearest-neighbor interaction dominates and the spins decouple into series of intercalated 1D Ising antiferromagnetic chains. That singular limit has 1D Ising universality for which $\gamma=1$. The finiteness of the HT series expansion results in a slow decay of $\gamma$ toward unity, as the large $\kappa$ terms in the series dominate the expansion. In this respect, the series is both a high-temperature and low- $\kappa$ expansion, which further restricts its range of validity.

\section{E. ANNNI roughening transition}

We first consider the impact of frustration on the roughening transition of the ANNNI model in the ferromagnetic regime (Fig. 6). Although the $T_{R}$ values extracted from simulations are quantitatively different from the series expansion results, ${ }^{109}$ similar trends are observed (Figs. 10). In particular, the transition temperature $T_{R}$ is relatively invariant to increases in frustration. The formation of an interface is surprisingly not aided by frustration, with $\mathrm{T}_{R}$ decreasing with increasing $\kappa$. And contrary to the scenario predicted for other microphaseforming systems, ${ }^{121}$ the roughening transition does not pass through or near the Lifshitz point. Instead, the roughening transition line on the $T-\kappa$ phase diagram is expected to reach the $\mathrm{FM}$ phase boundary near $\kappa \simeq 0.43$. Interestingly, a finite-temperature intercept suggests that the FM transition may be markedly different above and below $T_{R}$.

It has also been suggested that a roughening transition might be observed for the modulated phases as well. ${ }^{110,121}$ For the ANNNI model, however, we find no indication of interfacial roughening, at least for two simple modulated phases: phase $\langle 2\rangle$ at $\kappa=0.8$ and phase $\langle 3\rangle$ at $\kappa=0.52$ (Fig. 11). For the latter, the interface location remains clearly defined at all $T<T_{c}$, even for the largest systems considered. It cannot be excluded that a divergence may be observed for much larger interfacial areas than those we studied. Yet the lattice is here at least an order of magnitude larger than the size necessary for detecting roughening in the ferromagnetic phase (Fig. 6). We venture to speculate that at least on a lattice, the persistence length of the lamellae might be very large, possibly infinite. If that were the case, the roughening of the modulated layers would then coincide with $T_{c}$. Further simulation and theoretical work are necessary to clarify the situation.

\section{F. Phase diagrams}

Detailed low- $T$ series expansion studies of the phase behavior around $\kappa=1 / 2$ conducted by Fisher et al ${ }^{72,115}$ suggest that a series of "simple phases" of the form $\left\langle 2^{j} 3\right\rangle$ spring out from the the multiphase point at $T=0$ and "mixed phases" generated by combinations of neighboring simple phases branch out at $T>0$. The temperatures accessible in simulations are relatively far from the regime of validity of this theory and thus, from this point of view, it is misleading to compare them directly. It is nonetheless interesting to note that the two approaches appear to converge for $T \lesssim 2$. 


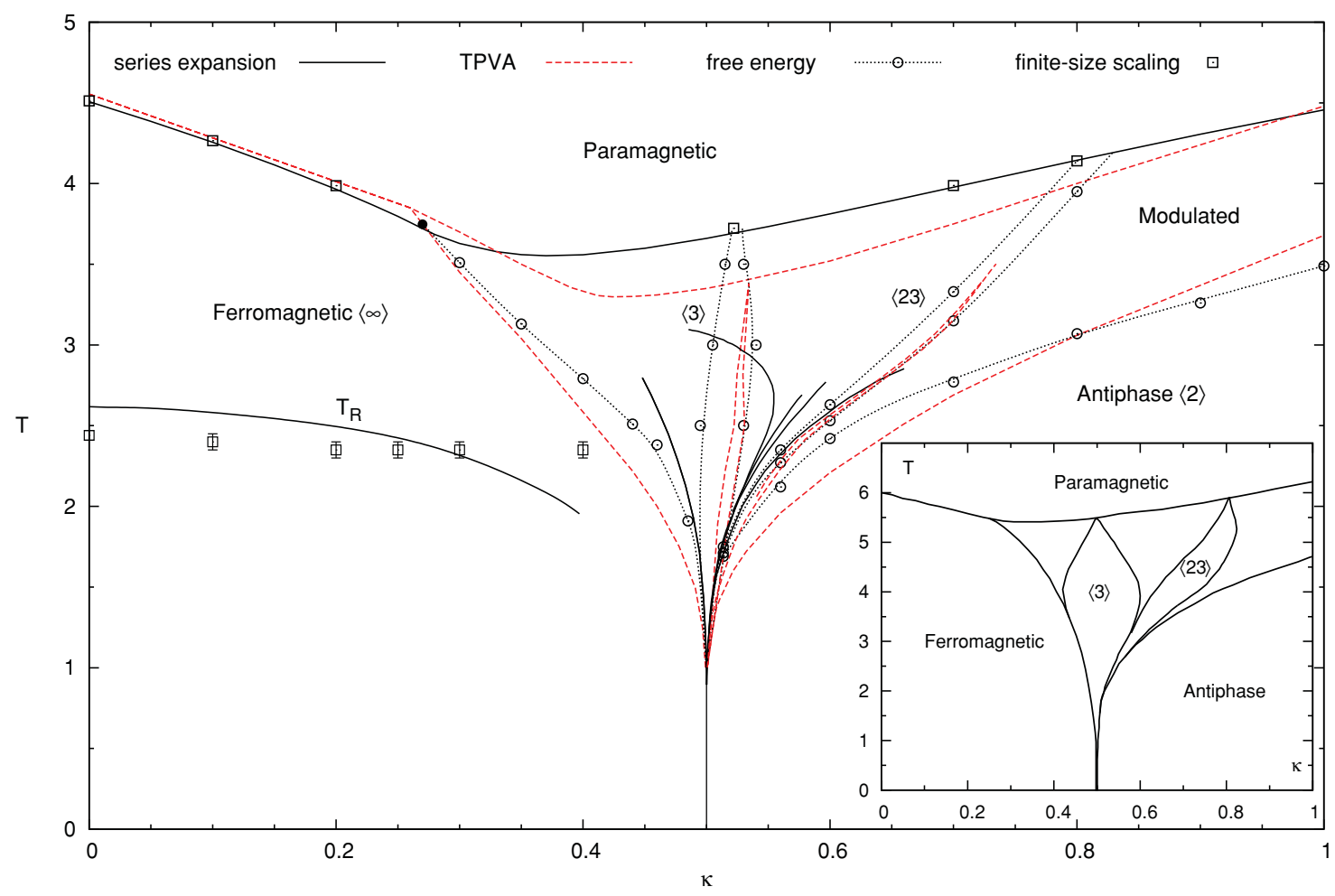

FIG. 10. (Color online) Phase boundaries for the ANNNI model obtained from $U_{4}^{*}(\square)$ and $F(\odot)$. The Lifshitz point location $(\bullet)$ is taken from Ref. 59. High- (Refs. 61,62) and low-temperature (Ref. 73) series expansions as well as TPVA (Ref. 76) results are represented. The stability wedges of phases $\langle 3\rangle$ and $\langle 23\rangle$ obtained from simulation are seen to be qualitatively different from the mean-field theory predictions (right inset; Ref. 55). The roughening transition results in the ferromagnetic regime are similar to the series expansion results (Ref. 109).

Various approximate theoretical treatments have been used to analyze the ANNNI phase diagram more globally. In addition to the traditional mean-field approach, ${ }^{74}$ an effective field $^{75}$ and a tensor product variational approach (TPVA) ${ }^{76}$ have more recently been used. These last two approaches reasonably capture the external boundaries of the modulated

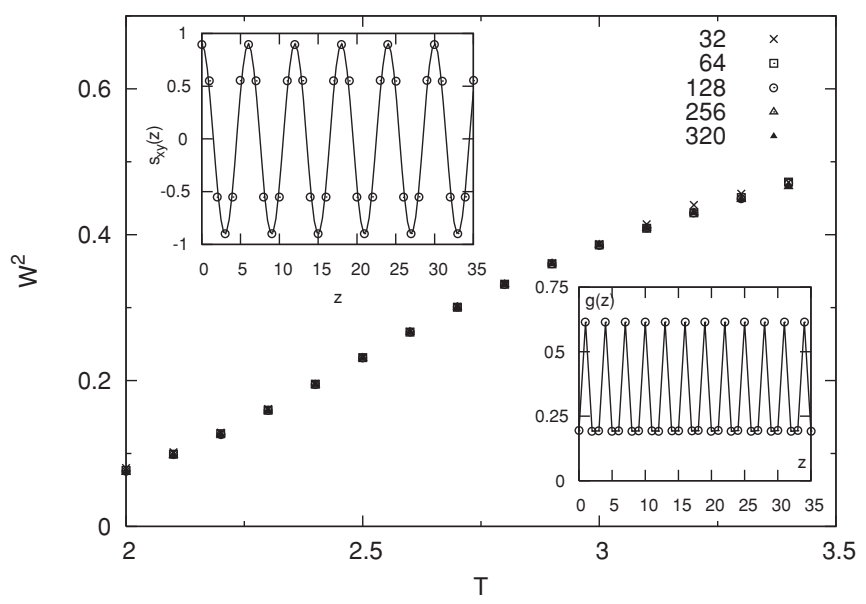

FIG. 11. Interface width $W^{2}$ of phase $\langle 3\rangle$ of the ANNNI model at $\kappa=0.52$ as a function of $T$ for various system sizes $L^{2} \times 36$. No roughening transition is detected within the stability regime. The magnetization profile $s_{x y}(z)$ for the system of $L=128$ at $T=3.0$ is also shown (left inset) along with its normalized gradient $g(z)$ (right inset). regime. The two treatments, however, qualitatively disagree on the internal structure of that same regime. On the one hand, the effective-field method, ${ }^{75}$ like the mean-field treatment and the soliton approximation, ${ }^{74}$ fills the modulated interior by exceptionally stable bulging simple phases, such as phase $\langle 3\rangle$ and phase $\langle 23\rangle$ (Fig. 10). On the other hand, TPVA predicts rather narrow stability wedges for the commensurate phases. ${ }^{76}$ The simulation results tend to favor the second scenario. Although the devil's staircase indicates that the rate of wave number change slows upon approaching $T_{c}$, only the antiphase has a broad presence in the modulated regime. The stability range of the different modulations is fairly small, and all of the phases commensurate with the periodic box are stable in turn. The branching and mixing of the stable low-temperature $\left\langle 2^{j} 3\right\rangle$ phases is already complete at the temperatures studied here. ${ }^{36,115}$ In particular, no special stability is observed for phases $\langle 3\rangle$ and $\langle 23\rangle$ (Fig. 10). For phase $\langle 3\rangle$ some bulging is seen, because of the slower rate of change of the periodicity near $\kappa=1 / 2$. Simulating larger lattices, which allow for a more refined $q$ selection, however, shrinks that phase's footprint. For phase $\langle 23\rangle$, the range of stability does increase slightly with $\kappa$, but the effect seems to be due to the finiteness of the lattice. In any case, the increase is much less pronounced than the bulging scenarios predict ${ }^{74,75}$ (Fig. 10).

For the IC model, qualitatively similar branching is expected in the devil's flower region, which is found between phases of integer periodicity. The systems simulated here are, however, too small to examine this issue critically. Except 
for the caveats presented in the previous sections, our results mostly agree with the simulation results of Ref. 43 .

\section{CONCLUSION}

Our simulation study has clarified the structure and transition properties of the modulated regime of the ANNNI and the IC models. Previous theoretical treatments had sometimes been insufficient, particularly concerning the stability regime of the various modulated phases, the critical nature of the ANNNI PM transition, and the role of roughening. The isotropic nature of the critical behavior of the ANNNI model beyond the Lifshitz point, despite the existence of anisotropic interactions and phases, is an interesting result that deserves future considerations. In the case of lamellar phase roughening, no clear conclusion can be drawn, but our results suggest that the phenomenon is at least a lot less pronounced than in the Ising model, which may give hope of experimentally forming microphase patterns on much larger scales than previously thought. From a theoretical perspective, it is also interesting to highlight, however, that mean-field theory is particularly adept at predicting the periodicity of modulated phases at the PM transition. This observation may explain why the approach has been so successful at describing order in other microphase-forming systems, such as diblock copolymers. $^{122,123}$

In addition to lamellar phases, modulated assemblies can exhibit a variety of other symmetries. They can also be observed off lattice. Generalizing the approach to continuous space and to other order types would thus greatly benefit the study of more complex microphase-forming systems. For the IC model, it could for instance help determine the nature of the modulated-Néel transition and other properties of the high- $Q$ regime, which we have only briefly explored. Completing the simulation tool set would also pave the way for studies of the nonequilibrium microphase assembly, where most of the materials challenges lie.

\section{ACKNOWLEDGMENTS}

We thank B. Mladek, J. Oitmaa, and M. Pleimling for their help at various stages of this project. We acknowledge ORAU and Duke startup funding.
${ }^{1}$ D. Chandler, Introduction to Modern Statistical Mechanics (Oxford University Press, New York, 1987).

${ }^{2}$ P. G. de Gennes, Scaling Concepts in Polymer Physics (Cornell University Press, Ithaca, 1979).

${ }^{3}$ G. Parisi and F. Zamponi, Rev. Mod. Phys. 82, 789 (2010).

${ }^{4}$ M. A. Glaser, G. M. Grason, R. D. Kamien, A. Kosmrlj, C. D. Santangelo, and P. Ziherl, Europhys. Lett. 78, 46004 (2007).

${ }^{5} \mathrm{M}$. Seul and D. Andelman, Science 267, 476 (1995).

${ }^{6}$ I. W. Hamley, The Physics of Block Copolymers (Oxford University Press, New York, 1998).

${ }^{7}$ L. Leibler, Macromolecules 13, 1602 (1980).

${ }^{8}$ S. A. Jenekhe and X. L. Chen, Science 283, 372 (1999).

${ }^{9}$ D. Wu, D. Chandler, and B. Smit, J. Phys. Chem. 96, 4077 (1992).

${ }^{10}$ G. Gompper and S. Zschocke, Phys. Rev. A 46, 4836 (1992).

${ }^{11}$ A. Stradner, H. Sedgwick, F. Cardinaux, W. C. K. Poon, S. U. Egelhaaf, and P. Schurtenberger, Nature (London) 432, 492 (2004).

${ }^{12}$ J. Rossatmignod, P. Burlet, H. Bartholin, O. Vogt, and R. Lagnier, J. Phys. C 13, 6381 (1980).

${ }^{13}$ M. Seul and R. Wolfe, Phys. Rev. Lett. 68, 2460 (1992).

${ }^{14}$ J. J. Sieber et al., Science 317, 1072 (2007).

${ }^{15}$ J. M. Tranquada, B. J. Sternlieb, J. D. Axe, Y. Nakamura, and S. Uchida, Nature (London) 375, 561 (1995).

${ }^{16}$ V. J. Emery, S. A. Kivelson, and J. M. Tranquada, Proc. Natl. Acad. Sci. USA 96, 15380 (1999).

${ }^{17}$ M. Vojta and S. Sachdev, Phys. Rev. Lett. 83, 3916 (1999).

${ }^{18}$ J. Orenstein and A. J. Millis, Science 288, 468 (2000).

${ }^{19}$ M. Vojta, Adv. Phys. 58, 699 (2009).

${ }^{20}$ C. V. Parker, P. Aynajian, E. H. D. Neto, A. Pushp, S. Ono, J. S. Wen, Z. J. Xu, G. D. Gu, and A. Yazdani, Nature (London) 468 , 677 (2010).

${ }^{21}$ T. Thurn-Albrecht, J. Schotter, C. A. Kastle, N. Emley, T. Shibauchi, L. Krusin-Elbaum, K. Guarini, C. T. Black, M. T. Tuominen, and T. P. Russell, Science 290, 2126 (2000).

${ }^{22}$ R. E. Cohen, P. L. Cheng, K. Douzinas, P. Kofinas, and C. V.
Berney, Macromolecules 23, 324 (1990).

${ }^{23}$ L. M. Leung and J. T. Koberstein, Macromolecules 19, 706 (1986).

${ }^{24}$ K. A. Koppi, M. Tirrell, and F. S. Bates, Phys. Rev. Lett. 70, 1449 (1993).

${ }^{25}$ P. Kofinas and R. E. Cohen, Macromolecules 27, 3002 (1994).

${ }^{26}$ S. A. Jenekhe and X. L. Chen, Science 279, 1903 (1998).

${ }^{27}$ L. Meli and T. P. Lodge, Macromolecules 42, 580 (2009).

${ }^{28}$ R. P. Sear and W. M. Gelbart, J. Chem. Phys. 110, 4582 (1999).

${ }^{29}$ D. Pini, J. L. Ge, A. Parola, and L. Reatto, Chem. Phys. Lett. 327, 209 (2000).

${ }^{30}$ J. L. Wu and J. S. Cao, Physica A 371, 249 (2006).

${ }^{31}$ A. J. Archer and N. B. Wilding, Phys. Rev. E 76, 031501 (2007).

${ }^{32}$ C. L. Klix, K. Murata, H. Tanaka, S. R. Williams, M. A., and C. P. Royall, e-print arXiv:0905.3393.

${ }^{33}$ C. L. Klix, C. P. Royall, and H. Tanaka, Phys. Rev. Lett. 104, 165702 (2010).

${ }^{34}$ J.-M. Bomont, J.-L. Bretonnet, and D. Costa, J. Chem. Phys. 132, 184508 (2010).

${ }^{35}$ R. Kretschmer and K. Binder, Z. Phys. B 34, 375 (1979).

${ }^{36}$ W. Selke, Phys. Rep. 170, 213 (1988).

${ }^{37} \mathrm{~J}$. Yeomans, in Solid State Physics-Advances in Research and Applications, edited by E. Henry and T. David, Vol. 41, (Academic, London, 1988), pp. 151-200.

${ }^{38} \mathrm{~W}$. Selke, in Phase Transitions and Critical Phenomena, edited by C. Domb and J. L. Lebowitz, Vol. 15 (Academic, London, 1992), pp. 1-72.

${ }^{39}$ B. Widom, J. Chem. Phys. 84, 6943 (1986).

${ }^{40}$ H. Fried and K. Binder, J. Chem. Phys. 94, 8349 (1991).

${ }^{41}$ M. W. Matsen, G. H. Griffiths, R. A. Wickham, and O. N. Vassiliev, J. Chem. Phys. 124, 024904 (2006).

${ }^{42}$ U. Löw, V. J. Emery, K. Fabricius, and S. A. Kivelson, Phys. Rev. Lett. 72, 1918 (1994). 
${ }^{43}$ M. Grousson, G. Tarjus, and P. Viot, Phys. Rev. E 64, 036109 (2001).

${ }^{44}$ P. M. Chaikin and T. C. Lubensky, Principles of Condensed Matter Physics (Cambridge University Press, New York, 1995).

${ }^{45}$ D. P. Landau and K. Binder, A Guide to Monte Carlo Simulations in Statistical Physics (Cambridge University Press, New York, 2000).

${ }^{46}$ M. E. Cates and S. T. Milner, Phys. Rev. Lett. 62, 1856 (1989).

${ }^{47}$ P. Charbonneau and D. R. Reichman, Phys. Rev. E 75, 050401 (2007).

${ }^{48}$ J. C. F. Toledano, F. Sciortino, and E. Zaccarelli, Soft Matter 5, 2390 (2009).

${ }^{49}$ U. Micka and K. Binder, Macromol. Theor. Simul. 4, 419 (1995).

${ }^{50}$ K. Zhang and P. Charbonneau, Phys. Rev. Lett. 104, 195703 (2010).

${ }^{51}$ W. Selke and M. E. Fisher, Phys. Rev. B 20, 257 (1979).

${ }^{52}$ R. J. Elliott, Phys. Rev. 124, 346 (1961).

${ }^{53}$ Y. Muraoka, T. Kasama, T. Shimamoto, K. Okada, and T. Idogaki, Phys. Rev. B 66, 064427 (2002).

${ }^{54}$ M. H. Jensen and P. Bak, Phys. Rev. B 27, 6853 (1983).

${ }^{55}$ W. Selke and P. M. Duxbury, Z. Phys. B 57, 49 (1984).

${ }^{56}$ R. M. Hornreich, M. Luban, and S. Shtrikman, Phys. Rev. Lett. 35, 1678 (1975).

${ }^{57}$ H. W. Diehl and M. Shpot, Phys. Rev. B 62, 12338 (2000).

${ }^{58}$ H. W. Diehl and M. Shpot, J. Phys. A 35, 6249 (2002).

${ }^{59}$ M. Pleimling and M. Henkel, Phys. Rev. Lett. 87, 125702 (2001).

${ }^{60}$ M. Henkel and M. Pleimling, Comput. Phys. Commun. 147, 419 (2002).

${ }^{61}$ S. Redner and H. E. Stanley, Phys. Rev. B 16, 4901 (1977).

${ }^{62}$ J. Oitmaa, J. Phys. A 18, 365 (1985).

${ }^{63}$ W. Selke and M. E. Fisher, J. Magn. Magn. Mater. 15/18, 403 (1980).

${ }^{64}$ E. B. Rasmussen and S. J. Knak Jensen, Phys. Rev. B 24, 2744 (1981).

${ }^{65}$ K. Kaski and W. Selke, Phys. Rev. B 31, 3128 (1985).

${ }^{66}$ F. Rotthaus and W. Selke, J. Phys. Soc. Jpn. 62, 378 (1993).

${ }^{67}$ W. Selke, Z. Phys. B 29, 133 (1978).

${ }^{68}$ T. Garel and P. Pfeuty, J. Phys. C 9, L245 (1976).

${ }^{69}$ M. Droz and M. D. Coutinho-Filho, AIP Conf. Proc. 29, 465 (1976).

${ }^{70}$ Z. Mo and M. Ferer, Phys. Rev. B 43, 10890 (1991).

${ }^{71}$ A. Michelson, Phys. Rev. B 16, 577 (1977).

${ }^{72}$ M. E. Fisher and W. Selke, Phys. Rev. Lett. 44, 1502 (1980).

${ }^{73}$ M. E. Fisher and W. Selke, Philos. Trans. R. Soc. London A 302, 1 (1981).

${ }^{74}$ P. Bak and J. von Boehm, Phys. Rev. B 21, 5297 (1980).

${ }^{75}$ A. Surda, Phys. Rev. B 69, 134116 (2004).

${ }^{76}$ A. Gendiar and T. Nishino, Phys. Rev. B 71, 024404 (2005).

${ }^{77}$ V. J. Emery and S. A. Kivelson, Physica C 209, 597 (1993).

${ }^{78}$ P. Viot and G. Tarjus, Europhys. Lett. 44, 423 (1998).

${ }^{79}$ M. Grousson, G. Tarjus, and P. Viot, Phys. Rev. E 62, 7781 (2000).

${ }^{80}$ A. Ciach and G. Stell, J. Chem. Phys. 114, 3617 (2001).

${ }^{81}$ S. C. Glotzer and A. Coniglio, Phys. Rev. E 50, 4241 (1994).

${ }^{82}$ C. B. Muratov, Phys. Rev. E 66, 066108 (2002).

${ }^{83}$ M. Tarzia and A. Coniglio, Phys. Rev. Lett. 96, 075702 (2006).

${ }^{84}$ M. Tarzia and A. Coniglio, Phys. Rev. E 75, 011410 (2007).

${ }^{85}$ R. Dickman and G. Stell, AIP Conf. Proc. 492, 225 (1999).
${ }^{86}$ G. Stell, in New Approaches to Problems in Liquid State Theory, edited by C. Caccamo, J.-P. Hansen, and G. Stell (Kluwer Academic Publishers, Dordrecht, 1999), pp. 71-89.

${ }^{87}$ A. Giuliani, J. L. Lebowitz, and E. H. Lieb, Phys. Rev. B 74, 064420 (2006).

${ }^{88}$ S. A. Brazovskii, Zh. Eksp. Teor. Fiz. 68, 175 (1975) [Sov. Phys. JETP 4185 (1975)].

${ }^{89}$ Z. Nussinov, J. Rudnick, S. A. Kivelson, and L. N. Chayes, Phys. Rev. Lett. 83, 472 (1999).

${ }^{90}$ N. G. Almarza and E. Enciso, Phys. Rev. E 64, 042501 (2001).

${ }^{91}$ J. G. Kirkwood, J. Chem. Phys. 3, 300 (1935).

${ }^{92}$ D. Frenkel and B. Smit, Understanding Molecular Simulation (Academic Press, London, 2002).

${ }^{93}$ M. Abramowitz and I. A. Stegun, Handbook of Mathematical Functions (Dover, New York, 1965).

${ }^{94}$ B. M. Mladek, P. Charbonneau, and D. Frenkel, Phys. Rev. Lett. 99, 235702 (2007).

${ }^{95}$ For the paramagnetic phase, where formally $B_{0}=0$, the integration is equivalently taken from finite $T$ to infinity.

${ }^{96}$ P. P. Ewald, Ann. Phys. 64, 253 (1921).

${ }^{97}$ A. M. Ferrenberg and D. P. Landau, Phys. Rev. B 44, 5081 (1991).

${ }^{98}$ M. E. J. Newman and G. T. Barkema, Monte Carlo Methods in Statistical Physics (Oxford University Press, New York, 1999).

${ }^{99}$ W. H. Press, B. P. Flannery, S. A. Teukolsky, and W. T. Vetterling, Numerical Recipes in C (Cambridge University Press, New York, 1992).

${ }^{100}$ K. Binder, Z. Phys. B 43, 119 (1981).

${ }^{101}$ K. Binder and J. S. Wang, J. Stat. Phys. 55, 87 (1989).

${ }^{102}$ For isotropic critical points, the universality of $U_{4}^{*}$ reduces to the aspect ratio of the system, as expected.

${ }^{103}$ A. P. Gottlob and M. Hasenbusch, Physica A 201, 593 (1993).

${ }^{104}$ N. Schultka and E. Manousakis, Phys. Rev. B 52, 7528 (1995).

${ }^{105}$ M. Campostrini, M. Hasenbusch, A. Pelissetto, P. Rossi, and E. Vicari, Phys. Rev. B 63, 214503 (2001).

${ }^{106}$ J. D. Weeks, G. H. Gilmer, and H. J. Leamy, Phys. Rev. Lett. 31, 549 (1973).

${ }^{107}$ R. H. Swendsen, Phys. Rev. B 15, 5421 (1977).

${ }^{108}$ K. K. Mon, D. P. Landau, and D. Stauffer, Phys. Rev. B 42, 545 (1990).

${ }^{109}$ A. Berera and B. Kahng, Phys. Rev. E 47, 2317 (1993).

${ }^{110}$ Y. Levin, Phys. Rev. Lett. 99, 228903 (2007).

${ }^{111}$ P. Bak, Rep. Prog. Phys. 45, 587 (1982).

${ }^{112}$ E. Burkner and D. Stauffer, Z. Phys. B 53, 241 (1983).

${ }^{113}$ P. Bak, Phys. Rev. Lett. 46, 791 (1981).

${ }^{114}$ A. K. Murtazaev and Z. G. Ibaev, Low Temp. Phys. 35, 792 (2009).

${ }^{115}$ M. E. Fisher and A. M. Szpilka, Phys. Rev. B 36, 5343 (1987).

${ }^{116}$ J. Adler, C. Holm, and W. Janke, Physica A 201, 581 (1993).

${ }^{117}$ M. Shpot and H. W. Diehl, Nucl. Phys. B 612, 340 (2001).

${ }^{118}$ S. Redner and H. E. Stanley, J. Phys. C 10, 4765 (1977).

${ }^{119}$ R. M. Hornreich, J. Magn. Magn. Mater. 15/18, 387 (1980).

${ }^{120}$ Y. H. Li and S. Teitel, Phys. Rev. B 40, 9122 (1989).

${ }^{121}$ B. Kahng, A. Berera, and K. A. Dawson, Phys. Rev. A 42, 6093 (1990).

${ }^{122}$ F. S. Bates and G. H. Fredrickson, Annu. Rev. Phys. Chem. 41, 525 (1990).

${ }^{123}$ F. S. Bates and G. H. Fredrickson, Phys. Today 52(2), 32 (1999). 